\title{
Movement tracking of cognitive processes: A tutorial using mousetrap
}

\author{
Dirk U. Wulff*,1,2, Pascal J. Kieslich",3 ${ }^{*, F e l i x ~ H e n n i n g e r ~}{ }^{3,4}$, Jonas M. B. Haslbeck ${ }^{5}$, and Michael \\ Schulte-Mecklenbeck ${ }^{2,6}$ \\ ${ }^{*}$ Authors contributed equally \\ ${ }^{1}$ University of Basel \\ ${ }^{2}$ MPI for Human Development \\ ${ }^{3}$ University of Mannheim \\ ${ }^{4}$ Ludwig Maximilian University of Munich \\ ${ }^{5}$ University of Amsterdam \\ ${ }^{6}$ University of Bern
}

\begin{abstract}
Movement tracking is a novel process tracing method promising unique access to the temporal dynamics of cognitive processes. The method involves high-resolution tracking of the hand or handheld devices, e.g., a computer mouse, while they are used to make a choice. In contrast to other process tracing methods, which mostly focus on information acquisition, movement tracking focuses on the processes of information integration and preference formation. In this article, we present a tutorial to movement tracking of cognitive processes with the mousetrap $\mathrm{R}$ package. We will address all steps of the research process from design to interpretation, with a particular focus on data processing and analysis. Using a representative working example, we will demonstrate how the various steps of movement tracking analysis can be implemented with mousetrap and provide thorough explanations on their theoretical background and interpretation. Finally, we present a list of recommendations to assist researchers in addressing their own research question using movement tracking of cognitive processes.
\end{abstract}

Keywords: Movement tracking, process tracing, cognitive processes, decision making

Movement tracking is a novel technique aimed at tracking the temporal development of cognitive processes. The technique involves the tracking of reaching movements of the hand using video cameras or movable devices (e.g., a computer mouse) while people choose between two or more spatially separated options. The result is a sequence of coordinates representing the movement's spatio-temporal development, a movement trajectory, which is assumed to reflect the cognitive processes that gave rise to the choice. Movement tracking has already been widely employed including for investigations into attention (e.g., Frisch et al., 2015; Miles

Dirk U. Wulff (D) https://orcid.org/0000-0002-4008-8022

Correspondence concerning this article should be addressed to Dirk U. Wulff, Department of Psychology, University of Basel, Missionsstrasse 60-62, 4055 Basel, Switzerland. E-mail: dirk.wulff@gmail.com

We are grateful to Laura Wiles for editing the manuscript. This work was supported by a grant from the Swiss Science Foundation (100015_197315) to Dirk U. Wulff. Pascal J. Kieslich and Felix Henninger were supported through a grant by the Deutsche Forschungsgemeinschaft (DFG, German Research Foundation) [KR2211/5-1] to Frauke Kreuter.
\& Proctor, 2015), decision making (e.g., Koop \& Johnson, 2013, Tabatabaeian et al., 2015), language (Blazej \& CohenGoldberg, 2015; Spivey et al., 2005), memory (Abney et al., 2015, Papesh \& Goldinger, 2012), numerical cognition (e.g., Faulkenberry \& Rey, 2014; Ganor-Stern \& Goldman, 2015), perception (e.g., Grosjean et al., 2012; van Vugt \& Cavanagh, 2012), and social cognition (e.g., Freeman et al., 2013, Martens et al., 2012). There are several excellent reviews summarizing this literature (Dotan et al., 2019, Faulkenberry et al., 2018; Freeman, 2018; Kieslich et al., 2019, Lopez et al., 2018; Stillerman \& Freeman, 2019; Stillman et al., 2018a, Tian \& Wu, 2019; Wulff et al., 2019).

In this article, we present a comprehensive tutorial on how movement tracking methodologies can be used to study cognitive processes. We will address the entire research process, starting from the implementation of movement tracking, to collecting, processing and analysing movement trajectories. We will discuss the advantages and disadvantages of various analysis approaches, provide practical recommendations for prospective users and, finally, discuss unresolved theoretical questions in movement tracking.

Throughout the article, we showcase the analysis of movement trajectories using our mousetrap package, currently the most comprehensive analysis package available for 
movement tracking 1 . The package, which has been build on the $\mathrm{R}$ programming language ( $\mathrm{R}$ Core Team, 2020), supports all steps of movement tracking data analysis, including raw data import from a wide array of formats, various algorithms for processing, analyzing and visualizing movement trajectories, as well as novel methodology for classifying movement trajectories types (Wulff et al., 2019). Where available, we will also point to other, complementary tools. Reproducible code for all analyses and figures presented in our tutorial can be accessed at https://pascalkieslich.github.io/mousetrapresources/mousetrap_tutorial.html.

Our article is structured as follows. First, we will discuss the promise of movement tracking for cognitive research, describe a representative movement tracking study that will serve as our working example throughout this article, and provide an overview of the elements of mousetrap. In the two major sections that follow, we will provide a detailed introduction to movement tracking processing and analysis. Finally, we will discuss practical and theoretical issues that researchers should be aware of when designing and interpreting movement tracking studies and provide a set of recommendations to guide future movement tracking research.

\section{The Promise of Movement Tracking}

Movement tracking promises unique insights into the cognitive process. Traditional process tracing techniques, such as eye-tracking (Russo, 2019), Mouselab (Willemsen \& Johnson, 2019), or Flashlight (Schulte-Mecklenbeck \& Huber, 2003) closely track the processes of information acquisition, but only indirectly shed light on the processes of information integration and preference formation (see also Schulte-Mecklenbeck et al., 2017, Wulff \& Hertwig, 2019). Movement tracking can be employed to track information acquisition, but its main purpose is to track the processes of preference formation. Movement tracking achieves this is two ways. First, by using choice tasks that have low requirements for information acquisition, so that behavior depends mostly on how information is processed while making a choice. Second, by recruiting higher temporal resolution than other process tracing methods (Schulte-Mecklenbeck et al., 2017), such that a detailed picture of preference formation is revealed.

As with other process tracing techniques, the usefulness of movement tracking rests on key assumptions about the mapping of the cognitive process onto the recorded process data, i.e., the movement trajectory. It makes the assumption that the current state of preference with regard to the available options influences key features of the movement, for instance, its angle or speed. Support for this assumption comes from neuro-psychological studies that have revealed close neuronal links between ongoing cognitive processes and motor output (Cisek \& Kalaska, 2005; Gold \& Shadlen, 2001 ) which suggest that the development of preference for- mation can affect the ongoing motor output as a choice is executed. How exactly the processes of preference formation impact the movement, however, is still an open question. Early applications of movement tracking worked under the assumption of a fully continuous mapping, where a continuous evidence accumulation process (Bogacz et al., 2006. Busemeyer et al., 2019; Lamberts, 2000) would directly control either the position or the angle of the movement throughout the movement (Spivey et al., 2005). Recent results, however, point towards a more discrete mapping, where the cognitive process influences the movement intermittently during the decision process (Wulff et al., 2019).

Crucially, the usefulness of movement tracking as a process tracing method only depends on that there is a link between cognitive process and movement, not whether the mapping is continuous or discrete. In either scenario, movement tracking provides valuable information on the development of the preference formation process, which can be used in at least three different ways. First, the trajectory of the movement can shed light on the relative strength of preference for the options, or, in other words, the degree of choice competition. Second, the movement trajectory can be used to identify the time point at which the preference formation process commits to a particular option, and in some case the order of commitment, including the detection of changes-ofmind (Wulff et al., 2019). Third, by varying features of the individual choice problems, movement trajectories can reveal at what point these features are considered during preference formation (e.g., Sullivan et al., 2015).

Next, we will introduce a working example that we will use throughout this article to showcase the potential of movement tracking for cognitive research. As many other movement tracking studies, it involves a forced choice between two options, which is known as the two-alternative, forcedchoice paradigm. Much of movement tracking theory and analysis have been developed with this paradigm in mind, however, this does not imply that the promise of movement tracking would be limited to this paradigm. Movement tracking has also been successfully applied to other paradigms involving more than two options (e.g., Koop \& Johnson, 2011; Moher \& Song, 2014), responses in surveys (FernándezFontelo et al., 2021; Horwitz et al., 2017; McKinstry et al., 2008), or even sensi-motor localization experiments (Fuchs et al., 2020).

\section{Tracking Semantic Categorization: A Working Example}

As our working example throughout this article, we use a replication of an early mouse-tracking study by Dale et al. (2007). Dale and colleagues sought to shed light on the temporal dynamics of semantic categorization. Their task

\footnotetext{
${ }^{1}$ For detailed documentation on mousetrap visit http://pascalkieslich.github.io/mousetrap/
} 


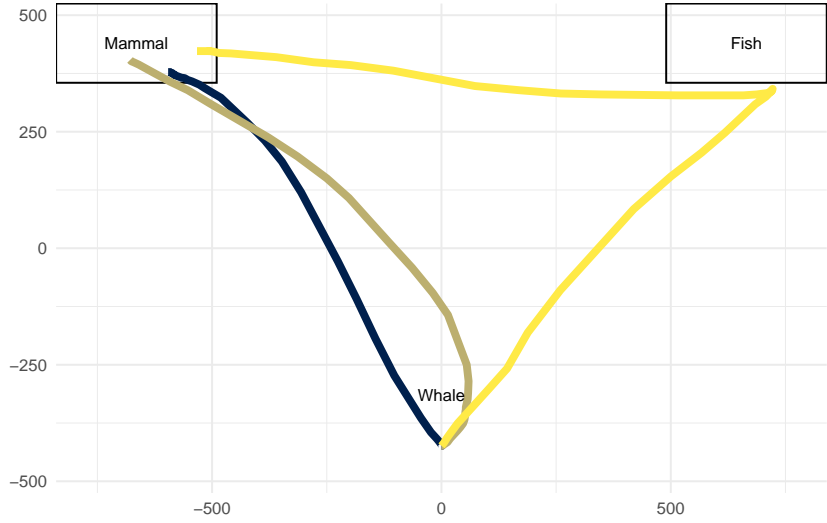

Figure 1

Illustration of the animal categorization task showing three exemplary mouse movement trajectories for a trial involving the categorization of "Whale" as either "Mammal" or "Fish".

required participants to select the correct taxonomic class of animals that varied in their typicality for their respective classes. For instance, in the case of an atypical trial, participants would be presented with the word "whale" and then asked to classify it as either "fish" or "mammal". Dale and colleagues hypothesized that the typicality of an animal should influence the cognitive dynamics underlying the choice in ways that could be made visible within the movement executing the choice. Specifically, they assumed that atypical items compared to items with more typical category members result in more response competition and therefore in trajectories that are attracted more strongly by the nonchosen option.

To test how typicality influences the cognitive process, Dale et al. (2007) used a procedure that has become a standard for movement tracking studies: Participants initiated a trial by clicking a start button located at the bottom center of the screen. The click triggered the display of the animal word, the critical piece of information, slightly above the start button. Participants then moved their mouse (their hand) upwards to select one of two response options located at the top left and right corners of the screen (i.e., one of the animal categories). Figure 1 illustrates this setup along with three characteristic movement trajectories from our replication study.

Our replication of this experiment (Kieslich \& Henninger, 2017) tested 60 individuals using the same 19 stimuli used by Dale et al. (2007) with an identical setup and procedure (translated into German; additional details regarding the experimental procedure and sample can be found in Kieslich \& Henninger, 2017). The raw data are available at https://pascalkieslich.github.io/mousetrap-resources/ and are also included in the mousetrap $\mathrm{R}$ package. The study was implemented using the OpenSesame experiment builder
(Mathôt et al., 2012) and the mousetrap-os plugin (Kieslich \& Henninger, 2017) for mouse-tracking.

\section{The mousetrap Suite}

Before we begin our movement tracking tutorial in the next section, we give a short overview over our mousetrap $\mathrm{R}$ package. The package is part of the mousetrap movement tracking suite (see Figure 2) that also includes mousetrap-OS (Kieslich \& Henninger, 2017) and mousetrap-web (Henninger \& Kieslich, in press), two software solutions for the implementation of movement tracking studies. The former, mousetap-OS, is a plugin for the Python-based experiment builder OpenSesame (Mathôt et al., 2012), whereas as the latter, mousetap-web, is a plugin for the JavaScript-based, online experiment builder lab.js (Henninger et al., 2021). More information about these companion solutions including software templates is available at https://pascalkieslich.github.io/mousetrap-resources/

The mousetrap $\mathrm{R}$ package provides a complete set of functions for processing and analyzing movement tracking data (for an overview of all functions see http://pascalkieslich.github.io/mousetrap/). In terms of processing, it can read in data from various formats, including the formats exported from OpenSesame and MouseTracker (Freeman \& Ambady, 2010, another software solution to implement movement tracking studies using the computer mouse). Once data are imported into the mousetrap object class, it offers functions to align the positions of movement trajectories, to resample data, to standardize trajectories, and, finally, to inspect and clean data, ensuring that only meaningful trajectories enter the analysis stage.

In terms of analysis, the mousetrap $\mathrm{R}$ package includes functions to calculate a wide range of descriptive properties, such as speed or direction, and summary statistics such as trajectory curvature or complexity. Further, it includes functions to visualize and evaluate trajectories. Finally, it includes functions for two advanced analytic pathways focusing on either trajectory dynamics or types.

The next two sections present the use of these mousetrap functions for the processing and analysis of movement tracking data, including necessary theoretical background, applications using our working example, and reproducible analysis code.

\section{Processing Movement Trajectories}

The first step of working with movement tracking data is to prepare the data for analysis. As with other processing tracing techniques, this can involve several steps and decisions that can be critical for the results. In this section, we discuss these steps, beginning with importing movement tracking data and continuing with various processing options concerning the adjustment of the trajectories' spatial layout and the resampling of the trajectory data. 


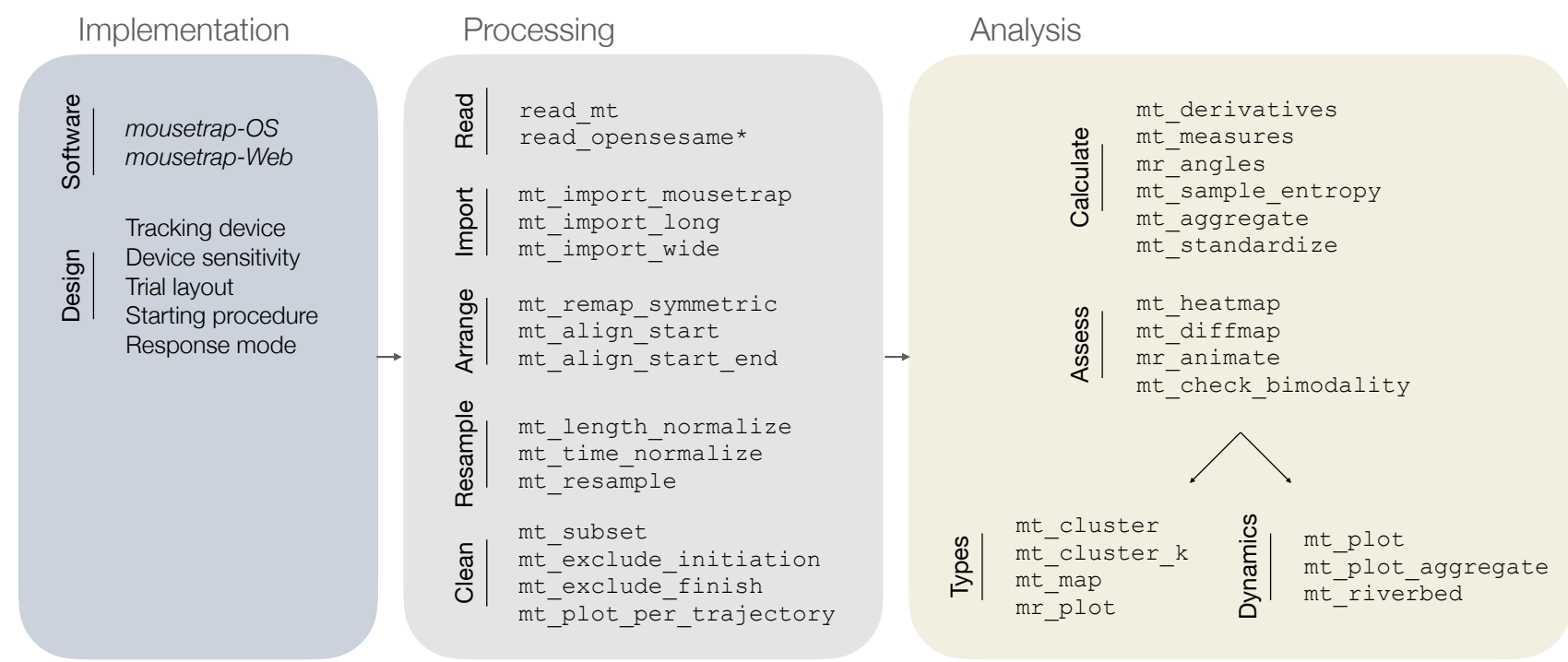

\section{Figure 2}

Overview over the mousetrap suite. The Implementation panel lists two mousetrap-related tools, mousetrap-OS and mousetrap-Web, that can be used to implement movement tracking on local machines or via the Web, respectively. It also lists important design dimensions discussed in a later section. The Processing and Analysis panels show the key functions of the mousetrap R package.

\section{Importing Movement Data}

Movement tracking records the $\mathrm{x}-, \mathrm{y}-$ (and potentially $\mathrm{z}-$ ) coordinate of the hand or a recording device at fixed intervals. As the time between position measurements is usually very short (e.g., $10 \mathrm{~ms}$ ), movement tracking datasets are often large, with up to several thousand coordinate pairs (or tuples) per trajectory, for each of potentially hundreds of trajectories per individual. Movement trajectories furthermore vary in length due to differences in decision time. For instance, in the case of our working example, the data encompasses for each of the 60 participants 19 trajectories that are between 72 and 2159 observations long, ranging between 703 and 21577 milliseconds.

Trajectory data is usually represented in one of two formats. The wide data format represents trajectories horizontally across the columns of a rectangular matrix or table, leaving empty cells at the end of rows representing shorter trajectories. The long data format represents trajectories across rows of a narrow matrix, with coordinate pairs and trajectories stacked on top of each other, implying that a trajectory identifier must be added as an additional column. Both formats are used in the analysis of movement tracking data. However, often a wide data representation is more economical after the processing of trajectories since this usually involves resampling trajectories to a common number of data points (see below).

\section{Importing Data using mousetrap}

Before movement trajectory data can be processed with mousetrap, the raw data must be read into $\mathrm{R}$ using one of many available functions for generic data formats (see, e.g., the rio package that bundles functions for importing many common file formats) or specialized functions such as mousetrap's read_mt for MouseTracker files or readbulk's read_opensesame for mousetrap-os generated OpenSesame files. Once data is read in, the next step is to transform it into the mousetrap object class using one of the functions named mt_import_*, where * represents one of long or wide depending on the format of the raw data. For data that has been collected using mousetrap-os, there is also a dedicated mt_import_mousetrap function.

The resulting mousetrap object is a list of two elements. The first element is a data frame called data with one row per trial that contains further information for each trajectory. In our working example, this includes the variables subject_nr, identifying the participant, Condition, coding whether the animal is typical or not, correct coding whether the choice was correct, and mt_id, a unique trial identifier added by mousetrap that is also used for the row names of data. The second element is a three-dimensional array called trajectories containing the trajectory data in a wide format. The array's first dimension has $N$ entries and distinguishes the different trajectories, the second dimension distinguishes the up to $M$ points used to represent the trajectories, and the third distinguishes the trajectory features, 


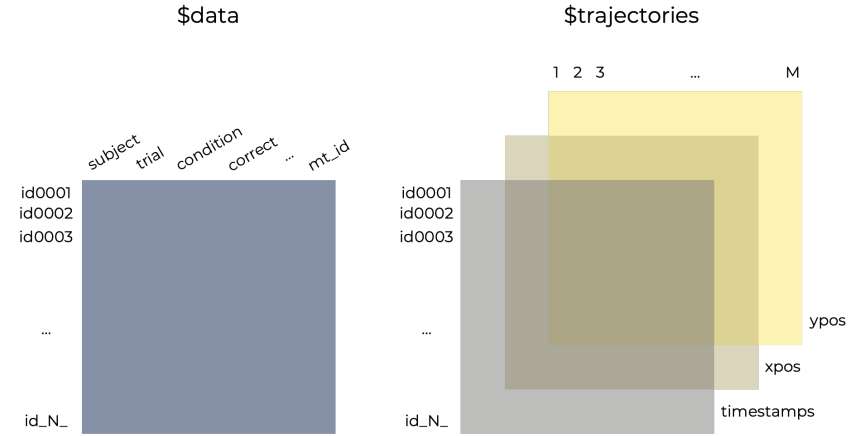

Figure 3

The two core elements of the mousetrap object. The rectangle on the left hand side represents the data element of the mousetrap object, the rectangles on the right-hand side the trajectories element.

including usually time stamps (timestamps), x-positions (xpos), and y-positions (ypos). As with the data object, the the first dimension of trajectories is named according to the mt_id variable. See Figure 3 for an illustration.

The code below shows how trajectory data from different file types can be read-in and imported into mousetrap. The first chunk loads the mousetrap library, which is necessary to be able to run all further processing and analyses. The second chunk reads in data from a standard comma separated file (CSV) and then shows how the readin raw data can be imported with either mt_import_wide or mt_import_long depending on the format of the raw data. If the raw data is stored in a long format, it is necessary to use the mt_id_label argument to provide the names of the variables that uniquely identify each trajectory. The third chunk makes use of readbulk's read_opensesame function to read in multiple mousetrap-os files from a folder and then imports them with the dedicated mt_import_mousetrap function. Finally, the fourth chunk directly uses the KH2017_raw data set, which contains the data from our working example and is already included in mousetrap, along with a processed version of the data set named KH2O17. The chunk first eliminates trials with incorrect answers, using the subset function, which is done to follow the analytic choices made by Dale et al. (2007). Then it imports KH2017_raw using mt_import_mousetrap. The resulting mt_data object will be used in all subsequent code sections.

\section{\# 1) Load mousetrap ------}

library (mousetrap)

\# 2) CSV raw data ------

\# Read raw data

library (readr)

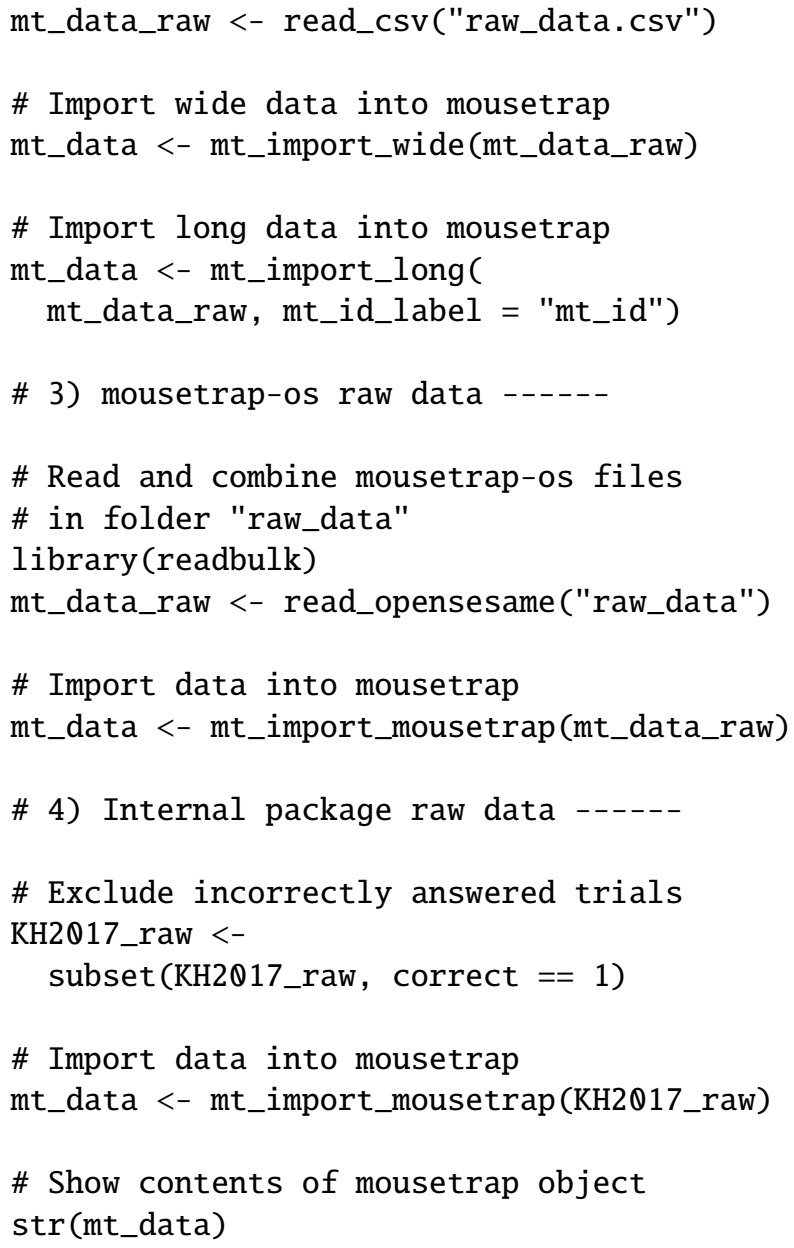

\section{Transforming Movement Trajectories}

Before movement tracking data can be analyzed, the movement trajectories typically must be transformed in order to make them comparable. The two most common transformation steps are the adjustment of the trajectories' spatial arrangement and the resampling and interpolation of trajectories.

\section{Adjusting the Trajectories' Spatial Arrangement}

In most movement tracking studies, including our working example, trajectories start in a common area, usually the bottom center of the screen, and end in an area belonging to one of the response buttons, usually the upper left and right corners (see Figure 11). A frequent first step in processing movement trajectories is therefore to remap trajectories such that all trajectories end on the same side, which simplifies later visualization and analysis (see Figures $4 \mathrm{~A}$ and $4 \mathrm{~B}$ for an illustration). This is possible, as typically the side of the choice is not of interest due to counterbalancing of the stimulus material. However, some analyses (e.g., Scherbaum et al., 2010) require that the side of the choice is preserved, in 
which case the researcher should either refrain from remapping or include the side of the choice as a variable in the data set. A frequent second step is to align the trajectories' start or end positions. This is done because start and end positions of the trajectories might still vary slightly across trials, for instance, because participants click different locations within the start and response buttons (e.g., Dale et al., 2007). Trajectories can also be aligned at both start and end positions; however, one should be aware that this implies stretching or compressing the dimensions of the trajectory. Most frequently, trajectories are aligned with regard to their start position by setting it to the point 0,0 so that trajectory coordinates can be interpreted as distances relative to the start position.

A third, less frequent, preprocessing step concerning spatial arrangement is the projection of trajectories into a new coordinate system. The MouseTracker software (Freeman \& Ambady, 2010), for example, automatically projects trajectories into a coordinate system with $\mathrm{x}$-values ranging from -1 to 1 and $y$-values ranging from 0 to 1.5 , with the point $[0,0]$ as the start position. However, mapping trajectories to a new coordinate system can affect the trajectories' aspect ratio and, with that, the relative importance of the trajectories' dimensions in the results. For instance, making a coordinate system narrower emphasizes the importance of the vertical relative to the horizontal dimension. Moreover, such projections usually lose the exact pixel information of the experimental setup.

\section{Resampling Trajectories}

Given that sampling rates are usually (more or less) constant, trials with different durations result in trajectories of different lengths. For example, in a setup with a $100 \mathrm{~Hz}$ sampling rate trials lasting $1 \mathrm{~s}$ and $2 \mathrm{~s}$ will result in 101 and 201 recorded positions, respectively. This presents a problem for many analyses that require trajectories to be represented by an equal number of points. To address this, one of two resampling methods is commonly employed.

Time-normalization (e.g., Spivey et al., 2005) interpolates trajectories such that they are represented by exactly the same number $(N)$ of temporally equidistant data points (see Figure 4 C for an illustration). $N$ is typically chosen to be 101 , following the early work by Spivey et al. (2005), who first introduced this resampling method. This value may represent a reasonable trade-off between retaining sufficient detail and enabling efficient processing; however, other values of $N$ may be justified depending on the length and complexity of trajectories. Time-normalization is typically used to facilitate analyses of the temporal development of trajectories (see Section Temporal Evolution of the Trajectory).

Length-normalization interpolates the trajectory into $N$ spatially equidistant data points or, in other words, $\mathrm{N}$ 1 equally long movement segments. Compared to time- normalization, in which the beginning and end of a trajectory are usually represented with higher resolution due to lower movement velocity, length-normalization is spatially uniform, and thus constant in spatial resolution throughout the trial. Length-normalization thereby helps emphasize the shape of the trajectory irrespective of the movement speed throughout the trial (see Figure 4D). For this reason, lengthnormalization is typically used in the detection of trajectory types (Wulff et al., 2019, see Sections The Bottom-Up Approach to Trajectory Types and The Top-Down Approach to Trajectory Types).

Finally, neutral resampling interpolates the trajectory such that timestamps are equidistant from each other, which is relevant only when for data that was recorded using variable sampling rates. One domain where neutral resampling is frequently employed is web-assessments, where sampling rates may vary across time and the computers running the study (Henninger \& Kieslich, in press).

\section{Preparing Trajectories using mousetrap}

Mousetrap provides functions for each of the spatial and temporal processing operations discussed above: mt_remap_symmetric maps trajectories to a common side, mt_align_start and mt_align_start_end align trajectories at the start and/or end, mt_time_normalize timenormalizes trajectories, and mt_length_normalize lengthnormalizes trajectories. By default, each of these functions uses the standard trajectories element in the mousetrap object (which initially contains the raw trajectories). However, via the use argument, functions can be instructed to use alternative trajectory representations.

In terms of storing the processed trajectories, the spatial transformation functions, such as mt_remap_symmetric and mt_align_start, by default overwrite the standard trajectories element. This is done because such transformations usually apply to all further analyses. The resampling functions, i.e., mt_time_normalize and mt_length_normalize, on the other hand, by default create new trajectory arrays in the mousetrap object so that users can later decide which trajectory representation to use in a given analysis. Either default can be overruled using the save_as argument, which allows users to specify how any of the processing functions store their results within the mousetrap object.

The code chunk below shows how to prepare trajectory data using mousetrap. The first chunk remaps all trajectories to the left-hand side (default) and then aligns the trajectories' start positions at the point $[0,0]$. The second chunk then adds new trajectory representations to the mousetrap object as separate elements. Specifically, the mt_time_normalize function adds the tn_trajectories element containing time-normalized trajectories, and the mt_length_normalize function adds the 
A $_{500}$

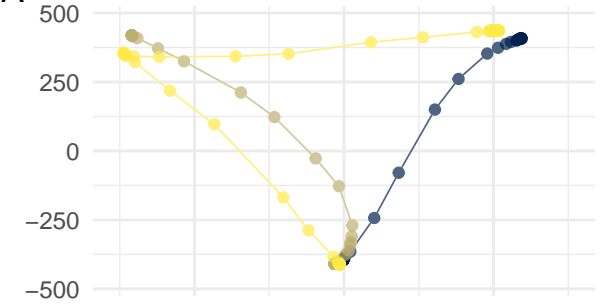

C

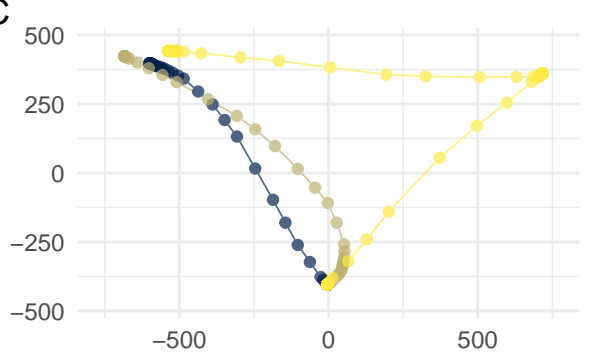

B

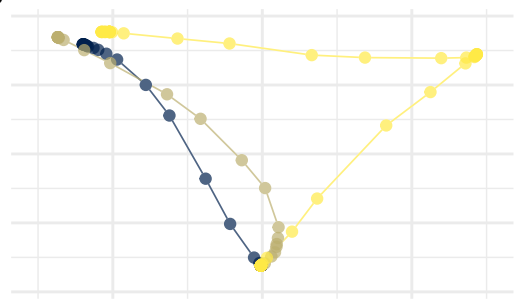

$\mathrm{D}$

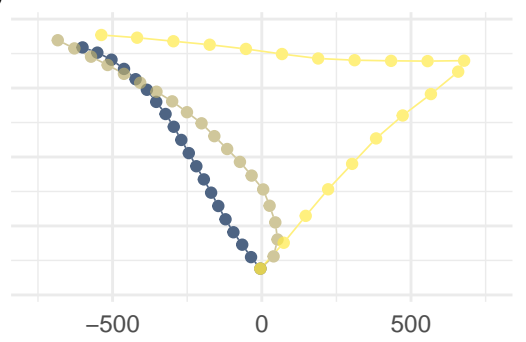

Figure 4

Common trajectory processing steps. Panel A shows three trajectories as they were originally recorded. Panel B shows the trajectories all mapped to the left side and aligned at the start position. Panel $C$ shows the trajectories after time-normalization. Panel D shows the trajectories after length-normalization.

In_trajectories element containing length-normalized trajectories. The final line shows the contents of the mousetrap object, which at this point should contain the elements data, trajectories, tn_trajectories, and ln_trajectories.

\# 1) Adjust the trajectories' spatial arrangement

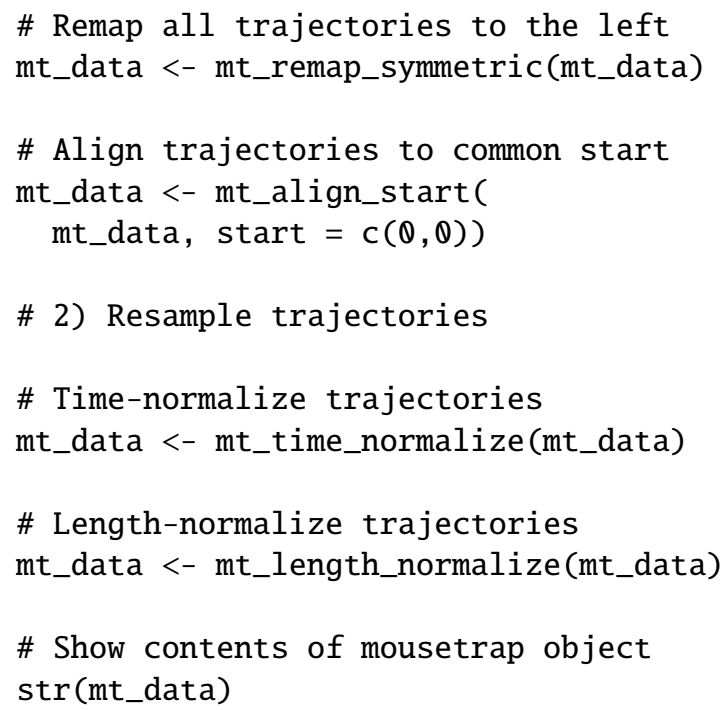

\section{Filtering movement trajectories}

In movement tracking, it is essential that the process of interest is appropriately reflected in all movement trajectories used in the analysis. In order to ensure this, several different trajectory filtering procedures can be employed. First, if the study involves factually correct responses, trials representing an erroneous response can be removed (e.g., Scherbaum et al., 2010, Spivey et al., 2005). The typical argument for excluding these trajectories is that such trials must have resulted from a different, irrelevant cognitive process (particularly given the fairly easy tasks that are frequently used in movement tracking studies). In our working example, for instance, $93.3 \%$ of responses categorize the animal into the correct category. This leaves a small proportion $(6.7 \%)$ of incorrect categorizations, which could have resulted from lapses of attention or faulty movement execution, giving reason for excluding those trials.

Second, if the study imposes strict requirements for movement times (see section The Role of Study Design), trials exceeding these limits can be removed. In the case of movement initiation, the argument for exclusion is that participants might have completed (most of) the decision process before the movement was initiated. Indeed, evidence suggests that long initiation times are associated with more straight, ballistic movement towards one of the options, likely reflecting only the outcome of the cognitive process, rather than its development (Grage et al., 2019). Studies have used various cutoffs ranging from $300 \mathrm{~ms}$ (e.g., Dshemuchadse et al., 2013) to $750 \mathrm{~ms}$ (e.g., Martens et al., 2012). A similar argument has been made for total response time, with studies excluding trials exceeding a total response time limit, with previously used cutoffs ranging from two (e.g., 


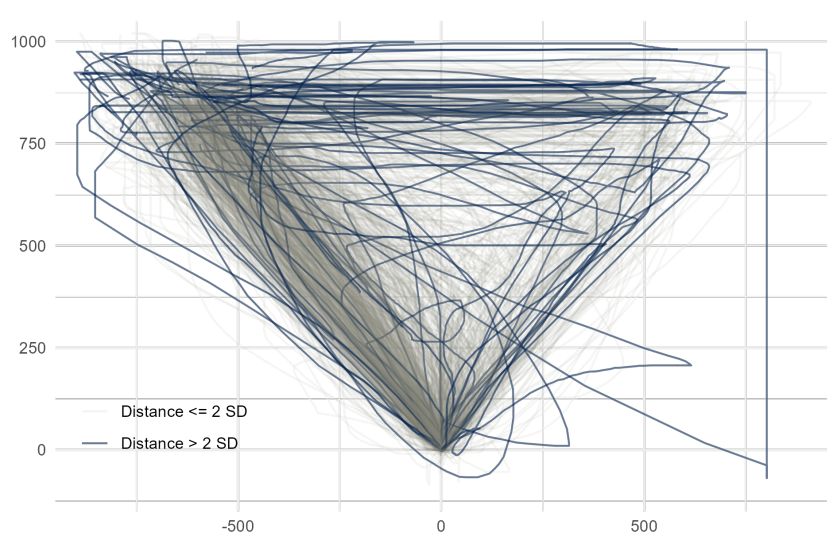

Figure 5

Outlier detection via prototyping. Figure shows, in blue, 34 trajectories from our working example that deviated more than two standard deviations from the respective closest trajectory prototype.

Quétard et al., 2015) to eight seconds (e.g., Martens et al., 2012).

Finally, trials with anomalous trajectories can be removed. For instance, Freeman et al. (2008) removed movements involving "erratic output producing non-interpretable looping cycling leftward and rightward" (p. 677). No precise criteria exist for defining anomalous trajectories, and most studies have relied on human judgment based on intuition and common sense. An alternative route to defining and identifying anomalous trajectories exists in analyzing trajectory similarities, as anomalous trajectories should, by definition, exhibit low similarity to most other trajectories. Figure 5 shows in blue potentially anomalous trajectories in our working example identified by comparing the trajectory's similarity to a set of trajectory prototypes (see section The Top-Down Approach to Trajectory Types).

\section{Trajectory Filtering with mousetrap}

mousetrap provides functions for trajectory filtering. The mt_subset function can be used for the selection of trials via logical comparisons. These comparisons are by default interpreted within the scope of the mousetrap data element (i.e., the specified variables are assumed to represent a column in data); however, via the check argument other elements can be specified.

The code below shows trajectory filtering using mousetrap. The first chunk selects trials where the column correct in data is of value 1 in order to only retain trials with correct responses. The second chunk maps trajectories to the standard prototypes (see section The Top-Down Approach to Trajectory Types). It then uses mt_standardize to determine the standardized distance of a trajectory to its closest prototype. Finally, it uses mt_plot to visualize anomalous trajectories ( $>2 S D$ ) and uses mt_subset to remove these cases. The third chunk uses mt_subset to remove individual trajectories (identified, e.g., through manual inspection) based on their id.

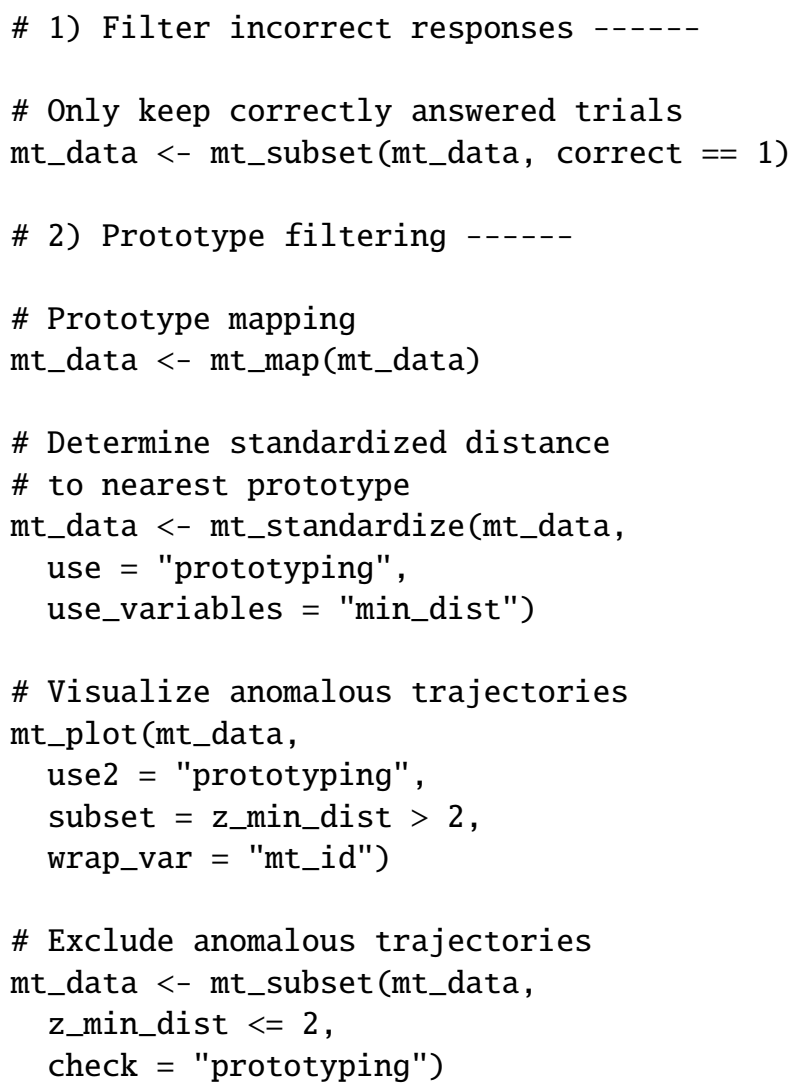

\# 3) Filter individual trajectories ------

\# Exclude trajectories by id

mt_data <- mt_subset (mt_data, !mt_id \%in\% c("id0426", "id0588"))

\section{Analysing Movement Trajectories}

In this section, we turn to the analysis of processed movement tracking data. We first discuss indices that summarize characteristics of trajectories using single values per trial. Such indices are the primary dependent variables in many movement tracking studies. We then present two alternative analysis strategies that harness more of the rich information contained in movement trajectory data: type-based analyses of the trajectories' spatial distribution and temporal analyses of the trajectories' development across time.

\section{Trajectory Indices}

Trajectory indices are trial-level summaries of one of three characteristics of movement trajectories: the trajectories' 
curvature, temporal development, and spatio-temporal complexity. Trajectory indices are often used as central dependent variables to capture the effect of experimental manipulations. For example, trajectory indices can be used to test the central hypothesis of our working example; namely, that trajectories in atypical trials should show more attraction to the non-chosen option than in typical trials.

\section{Curvature Indices}

Curvature indices capture the degree to which a trajectory is bent towards the non-chosen option. Curvature is commonly defined in reference to an idealized movement trajectory that directly connects the start and end points of the observed trajectory through a straight line (e.g., Freeman \& Ambady, 2010; Spivey et al., 2005). Specifically, curvature is quantified as the individual trajectory's deviation from this line in terms of the perpendicular distance of either a single or all points. Curvature indices are typically thought of as a direct measure of response competition. That is, strong curvature is thought to arise from relatively high levels of preference for the non-chosen alternative. Note that most curvature indices have been proposed with a two-dimensional, two-option scenario in mind, such as the one of our working example; however, it is straightforward to generalize them to more complex scenarios.

Among single-point measures, maximum absolute deviation $(M A D)^{2}$ is most frequently used. It is defined as the signed maximum absolute distance of the observed trajectory from the straight line (in either direction), where the sign indicates whether the distance is toward (positive) or away from (negative) the alternative. A related measure is the maximum deviation above the ideal trajectory $\left(M D_{a b o v e}\right)$, which (in contrast to $M A D$ ) only considers deviations in the direction of the non-chosen alternative (Kieslich \& Hilbig, 2014). Among measures that integrate deviations from the ideal line across all points on the trajectory, a common is the average deviation $(A D)$, which is defined as the arithmetic mean of all signed pointwise deviations between the trajectory and the ideal trajectory (Koop \& Johnson, 2013). A similar measure is the area under curve $(A U C)^{3}$ which typically is defined as the area between the observed and idealized trajectory (where areas below the idealized trajectory are subtracted) (e.g., Freeman \& Ambady, 2010, Spivey et al., 2005). This means that whereas $A D$ is sensitive to the temporal dynamics of the movement, $A U C$ is not, implying that the two can lead to different results.

Single-point and integrative curvature measures have different properties. Single-point measures are easier to interpret, but may not, due to their focus on a single point, be representative of the trajectory as a whole. Integrative measures weigh the deviation across the trajectory and are therefore more representative of its entirety; however, they give more weight to points at the start and end of the trajectories, and proportionally less to the more informative parts of the trajectory.

\section{Complexity Indices}

Complexity indices capture a trajectory's spatio-temporal degree of disorder (Hehman et al., 2015). The most frequently used complexity index is the number of flips, directional changes ${ }^{4}$ along the axis of interest, typically the horizontal axis (Freeman \& Ambady, 2010). A similar index is the number of reversals, defined as the number of times a trajectory crosses the midline between the options (Koop \& Johnson, 2013). A third index of complexity exists in the sample entropy of a dimension of interest (Dale et al., 2007; Hehman et al., 2015). Sample entropy is a standard complexity measure for time series data that measures their degree of self-similarity (see Yentes et al., 2013, for details).

Like trajectory curvature, complexity is thought to reflect response competition. For instance, Freeman and Ambady (2010) argue: "if both response alternatives simultaneously attract participants' mouse trajectories (relative to only one), this additional stress might manifest as less smooth, more complex, and fluctuating trajectories" (p. 230). Consistent with this view, manipulations of conflict tend to also produce differences in complexity indices with higher conflict being associated with higher complexity (Dale et al., 2007; Koop \& Johnson, 2013, McKinstry et al., 2008).

\section{Temporal Indices}

A third set of trial-level indices focuses on the temporal development of the movement. A first set of indices results from decomposing the overall response time, i.e., the time between the trial onset and the choice, into its components, motion time, defined as the time during which the movement is ongoing, and idle time, defined as the total time during which there is no movement (Cheng \& González-Vallejo, 2018). Idle time can be decomposed further into initiation time, defined as the idle time prior to the first onset of movement (Dale et al., 2007; Faulkenberry et al., 2015; Freeman \& Ambady, 2010), and motor pauses, defined as the remaining idle time after movement initiation (Calcagnì et al., 2017). A second set of temporal indices summarizes the velocity and acceleration profiles of the movement, such as maximum velocity or maximum acceleration (Koop \& Johnson, 2013).

\footnotetext{
${ }^{2}$ Also referred to as maximum deviation $(M D)$ in previous studies (e.g., Freeman \& Ambady, 2010, McKinstry et al., 2008)

${ }^{3}$ Note that there exist different implementations of $\overline{A U C}$ that can produce different results.

${ }^{4}$ Instead of counting every directional change as a flip, some studies specify a minimum distance that needs to be covered so that a change in direction is counted as flip. This method is implemented in mt_measures.
} 
Among temporal indices, initiation time has received most attention. Like curvature and complexity, it has been interpreted as a signal of response conflict (e.g., Dale et al., 2007). However, results concerning the relationship between response conflict and initiation time have been mixed. One reason for this could be study design, with some studies imposing a strict, short time limit on movement initiation (e.g., Faulkenberry et al., 2015) potentially leading to smaller sensitivity to competition than studies imposing longer or even no time limit at all (e.g., Incera \& McLennan, 2016). That is, it seems plausible that for initiation time to capture conflict, individuals must be given sufficient opportunity to vary in their initiation time. As these examples show, the information carried by time-related indices can hinge on the characteristics of the experimental implementation (Fischer \& Hartmann, 2014; Wirth et al., 2020).

\section{The Dimensionality of Trajectory Indices}

Although most trajectory indices have been linked to response competition, it has been suggested that they could provide a nuanced characterization of the underlying process. For instance, Freeman and Ambady (2010) suggested that single-point and integrative indices of curvature may capture maximum and overall response competition, respectively. Furthermore, it has been suggested that complexity indices may capture unique psychological elements beyond those captured by curvature indices. Koop and Johnson (2013), for instance, distinguished momentary valence (i.e., the current, continuous evaluation of each option) from absolute preference (i.e., the option with the higher valence) and associated changes in momentary valence with flips and changes in absolute preference with reversals. Similarly, complexity has been equated with wavering, and curvature with difficulty (Cheng \& González-Vallejo, 2018).

Notwithstanding these claims, empirical results typically suggest a high degree of overlap both within and between trajectory index classes. For instance, based on the data of our working example, we observed correlations among curvature indices between $r=.79$ (AUC x AD) and $r=.99$ (MAD $\left.\mathrm{x} M D_{\text {above }}\right)$, among complexity indices between $r=.49$ (Flips x Sampleentropy) and $r=.69$ (Flips x Reversals), and among temporal indices, excluding initiation time, between $r=.43$ (Motorpauses x Movement) and $r=.92$ (RT x Motorpauses), see Figure6A. Moreover, we observed substantial correlations between curvature and complexity (mean $r=.50$ ), curvature and temporal indices excluding initiation time (mean $r=.26$ ), as well as complexity and temporal indices excluding initiation time (mean $r=.37$ ). These correlations show a high to very high degree of overlap within indices of the same class, as well as substantial correlations between them. Only initiation time deviates from this pattern, likely due to its compensatory relationship with movement time, showing small negative correlations ranging from $r=-.11$ (Initiationtime $\mathrm{x}$ Motorpauses) to $r=-.21$ (Initiationtime x Sampleentropy) with all indices, except overall $R T$, which includes Initiationtime.

The inter-correlations between trajectory indices imply that their true dimensionality is much lower than the number of available indices. For our working example, a principle component analysis (excluding $R T$, to avoid a rankdeficient correlation matrix) revealed that $53 \%$ of variance can be explained using a single component, and $92 \%$ using five components. Additionally, all indices were sensitive to the manipulation of typicality (see Figure 6B) with Cohen's $d$ values ranging from .26 (Initiationtime) to $.60(A D)$. This means that, consistent with their use in the literature, all indices capture response competition, to a certain extent. However, this does not preclude the possibility that trajectory indices capture other and potentially unique aspects of the psychological process.

\section{Analyzing Trial-level Indices using mousetrap}

Most of the trajectory indices discussed above can be calculated using mousetrap's mt_measures function. By default, it uses the trajectories element; however, as in other functions, different trajectory elements can be specified via the use argument. The computed measures are stored in a new element called measures. Additional measures concerning velocity and acceleration are calculated if they were previously added using mt_derivatives. Finally, sample entropy can be added to measures using a dedicated mt_sample_entropy function.

To facilitate statistical comparisons, mousetrap further provides two functions for aggregating trajectory indices. The mt_aggregate function aggregates trajectory indices within condition, whereas the mt_aggregate_per_subject function does so within individuals (and conditions). To compare trajectory indices between conditions, studies would typically aggregate values first within individuals and then carry out comparisons using off-the-shelf statistical tests. However, it is generally preferable to analyse the dis-aggregated data on the trial level, while accounting for potential heterogeneity between individuals. This approach typically offers greater statistical power and permits flexible inclusion of additional covariates, for instance to account for item effects. Appropriate models are provided by the lme4 (Bates et al., 2015), afex (Singmann et al., 2015), or brms (Bürkner, 2017) packages.

The code below illustrates simple analyses that can be performed using trajectory indices. The first chunk uses the mt_measures function to compute indices, including MAD values. The next chunk carries out a subject-level analysis, with the mt_aggregate_per_subject function being used to aggregate MAD values (the name of the variable to aggregate is specified via the use_variables argument) per experimental condition and participant (speci- 


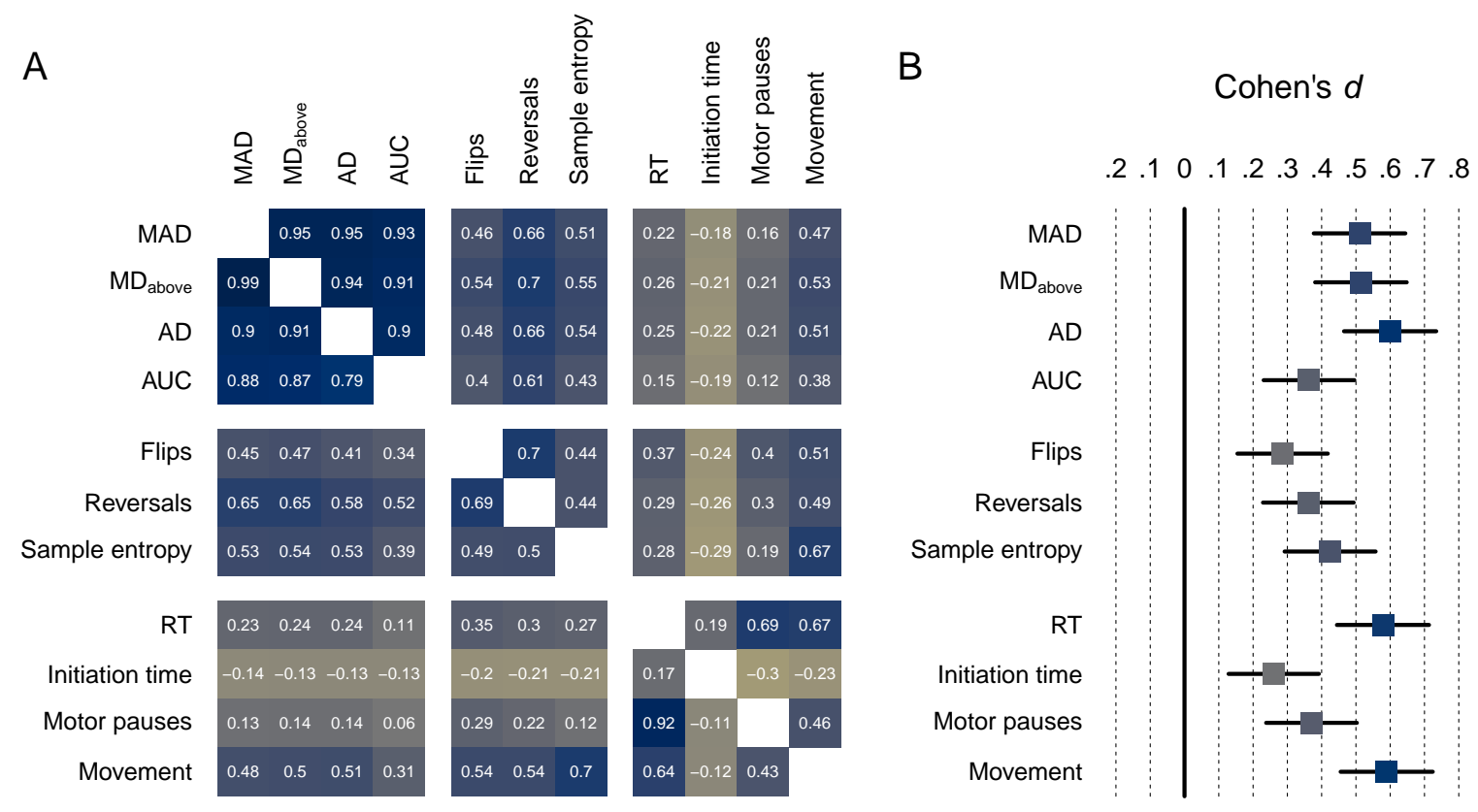

\section{Figure 6}

Overlap between trajectory indices. Panel A shows the Pearson (lower triangle) and Spearman (upper triangle) intercorrelations between the four curvature, three complexity, and four temporal indices discussed in the text for our working example. Panel B shows the indices' sensitivity to the typicality manipulation of our working example in terms of Cohen's $d$.

fied via the use2_variables and subject_id arguments respectively). The aggregated MAD values are then compared between conditions using the standard paired t-test.

To carry out analyses at the trial-level, the final chunk below first uses R's standard merge function to join the information contained in the grouping variable(s) in the data element with the trial-level indices in the measures element of the mousetrap object. Then it employs the lmer function of the lme4 package to run a mixed model predicting MAD.

\section{\# 1) Compute trajectory indices -...--}

mt_data <- mt_measures(mt_data)

\# 2) Subject-level analysis ------

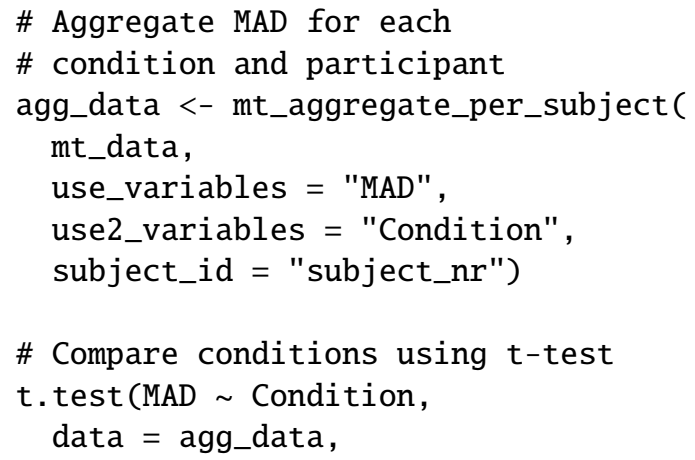

$$
\text { paired }=\text { TRUE) }
$$

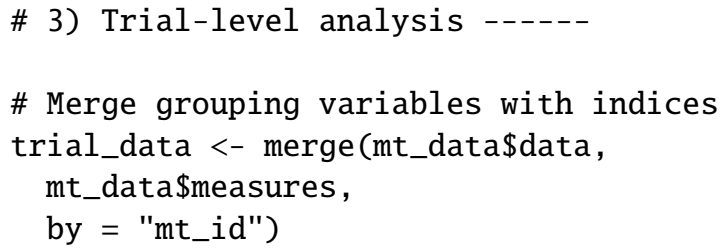

\# Compare MADs using linear-mixed model

\# including a random intercept per participant library (lme4)

lmer(MAD Condition + (1|subject_nr), data = trial_data)

\section{Advanced mouse- and hand-tracking analysis}

Despite the widespread use of trajectory indices in the literature, their ability to unveil the dynamics of the cognitive process is limited. First, the high correlations among indices largely preclude a fine-grained characterization of the movement and the underlying processes. Second, and more importantly, they compress the dynamic pattern within trajectories into a single number, thereby losing valuable information contained in the full movement trajectory.

In this section, we will discuss two advanced analysis approaches to movement-tracking that make use of the full tra- 
jectory data. One approach focuses on the spatial characteristics of trajectories, with the goal of uncovering distinct movement types that might correspond to distinct modes of the underlying cognitive process. The other focuses on the temporal characteristics of the trajectory, in order to shed light on the development of the cognitive process over time. Both approaches provide distinct insights into the cognitive process; however, they are based on different assumptions. Most temporal analyses assume that trajectories are homogeneous, whereas type-based approaches do not make this assumption and are thus more useful when this assumption is not met. As a result, researchers interested in applying either of the two approaches first should investigate the level of trajectory homogeneity or heterogeneity in the data, a question we will turn to next.

\section{Assessing Trajectory Homogeneity}

The traditional assumption underlying movement tracking is that the effect of response competition on movement trajectories is a direct and continuous one (Freeman \& Ambady, 2010, Spivey et al., 2005). However, it still is an open question whether and under which circumstances movement trajectories provide a continuous, high-resolution representation of the underlying process (Wulff et al., 2019). Movement tracking studies therefore must also consider the alternative scenario in which the cognitive process and movement trajectories map onto each other in a more discrete, intermittent way. For instance, Spivey et al. (2005), in one of the first movement tracking studies, considered as a rival hypothesis that trajectories could unfold in a ballistic fashion so as to give rise to two distinct types of trajectories: trajectories that move directly to the chosen option and trajectories that move all the way to the non-chosen option before reversing course to the eventually chosen option. The presence of such types poses problems for the idea that response competition expresses continuously into the movement. It also poses problems for analytic approaches that rely on aggregation. Specifically, when trajectories occur in distinct types, aggregate trajectories or aggregate trajectory indices are unlikely to be representative of the actual distribution of trajectories. Moreover, aggregate trajectories may create the incorrect impression of a continuous range of variation (e.g., of trajectory curvature) that is purely an artefact of aggregation.

To mitigate these potential problems, researchers can check for the presence of distinct trajectory types by inspecting the distribution of curvature indices (e.g., MAD or AUC) for bimodality, using, for instance, the bimodality coefficient (Pfister et al., 2013) or Hartigan's dip statistic (Hartigan \& Hartigan, 1985). This approach is based on the assumption that heterogeneity expresses in extremely different types of trajectories that produce distinct modes in the curvature distribution. However, the bimodality tests approach may not be as successful as often portrayed, in that bimodality tests may not be able to detect the presence of trajectory types (Pfister et al., 2013; Wulff et al., 2019): According to recent evidence (Kieslich et al., 2020, Lepora \& Pezzulo, 2015; Tomlinson Jr et al., 2013; Wulff et al., 2019), trajectories frequently occur in more than two types, which may not be easily distinguishable on the basis of trajectory indices. As a consequence, other approaches for assessing trajectory homogeneity are likely more suitable.

One simple and robust approach to detecting multiple types of trajectories is data visualization. Figure $7 \mathrm{~A}$ shows the distribution of raw trajectories for typical (yellow) and atypical (blue) trials in our working example (trajectories where the right option was chosen were remapped to the left option). The figure reveals two dominant types of trajectories: those that go straight to the chosen option and those that first visit the non-chosen option. Moreover, it shows a number of trajectories that fall between these prototypical trajectories, possibly suggesting additional types. This clearly suggests that, in our working example, Spivey et al. (2005)'s ballistic scenario has materialized. As a consequence, average trajectories for the two trial types would fall somewhere between the two dominant types and thereby fail to represent the empirical distributions of trajectories in either condition. Another consequence is that any difference in average trajectories, or average trajectory indices for that matter, may not reflect an average change concerning all trajectories, but simply a shift in the proportions of trajectory types. Figure $7 \mathrm{~B}$ shows a smoothed version of Figure 7A, highlighting local differences in trajectory distribution between conditions. The figure reveals that, indeed, atypical trials (in blue) have a higher density of trajectories in the region leading to, located around, and moving away from the non-chosen option, whereas typical trials have higher density on the direct path between the start and the chosen option. This strongly suggests that the two types of trials co-vary with the types of trajectories.

\section{Assessing Trajectory Homogeneity using mousetrap}

mousetrap provides functions for assessing trajectory homogeneity using bimodality tests and visualizations. The mt_check_bimodality function carries out two common bimodality tests, the bimodality coefficient (Pfister et al., 2013) and Hartigan's dip statistic (Hartigan \& Hartigan, 1985). The functions mt_plot and mt_heatmap offer ways to visualize the distribution of raw trajectories, as individual lines or overall density, respectively. As mt_plot is built on top of the widely-used ggplot2 package (Wickham, 2016), it can be customized and extended using various ggplot2 features, such as facets (see, e.g., Figure 12). The mt_heatmap function plots trajectories as a heatmap of trajectory densities, which gives a clearer picture of the distribution of trajectories and provides additional features, such as coloring according to a third trajectory dimension (e.g., ve- 
A

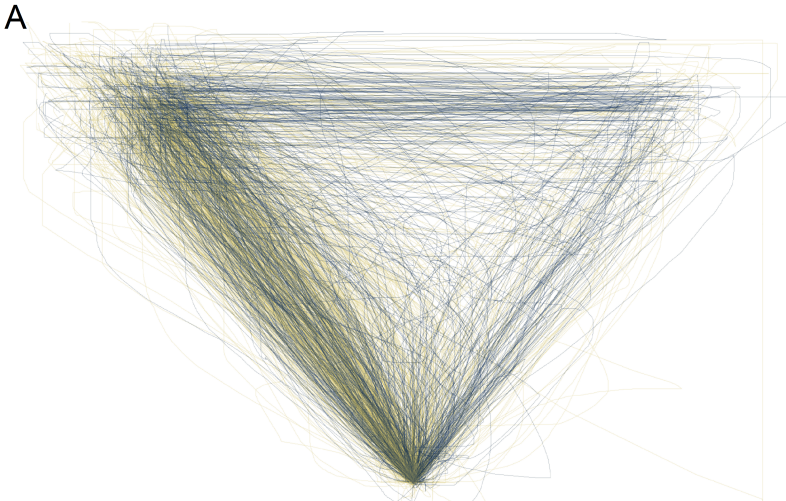

B

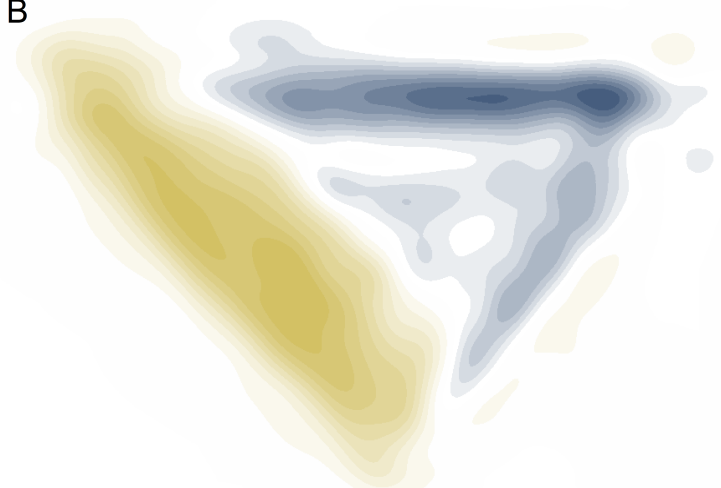

Figure 7

Assessment of trajectory homogeneity. Panel A shows the spatial distribution of trajectories of the atypical (blue) and typical (yellow) trajectories from our working example. Panel B shows the same trajectories with a high degree of smoothing highlighting the differences in trajectory distribution between conditions. Note that transparent regions reflecting equal density may or may not be populated by trajectory points, as can be gleaned from comparing the two panels.

locity), and smoothing. The mt_diffmap function provides a visual comparison of trajectory densities between conditions as shown in Figure 7. Finally, the mt_animate function creates animated visualizations of trajectories, which can further assist in identifying the kinds of trajectories contained in a data set.

The code below demonstrates how to create visualizations of movement tracking data that will help to assess trajectory homogeneity. The first line uses mt_plot to plot all trajectories in our working example in a single panel, coloring trajectories as a function of study condition. Setting alpha $=.1$ results in a low level of opacity, which helps distinguish overlaying trajectories. The second line uses mt_heatmap to create a heatmap based on the same data. In contrast to $m t \_p l o t, m t \_h e a t m a p$ automatically chooses an appropriate opacity. The third line creates a difference heatmap that illustrates differential point densities between the typical and atypical trials. The arguments smooth_radius $=10$ and $n \_s h a d e s=10$ imply a high level of smoothing and a small number of different color values, both of which can aid interpretation. Finally, mt_animate produces an animated visualization of all trajectory dat ${ }^{5}$.

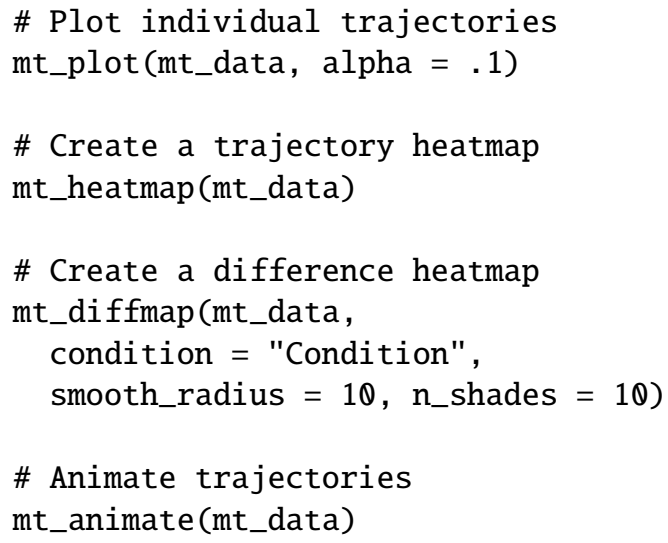

\section{The Bottom-Up Approach to Trajectory Types}

The analysis of movement trajectories as types separates trajectories into distinct trajectory groups. Two approaches exist to achieve this: the bottom-up approach based on cluster analysis and the top-down approach based on prototype matching (see Wulff et al., 2019).

The bottom-up approach uses cluster analysis to group trajectories into a predefined number of types. This typically involves two steps. First, the distance, a measure of (dis)similarity, is calculated between every pair of trajectories, for instance using the Euclidean or other distance functions. To facilitate this step, trajectories are typically lengthnormalized to place equal emphasis on all sections of the trajectory's shape while representing each trajectory with the same number of points (see section Resampling Trajectories). Second, the matrix of trajectory-pair distances is analyzed by a clustering algorithm, such as k-means or hierarchical clustering, which will group the trajectories into a predetermined number of clusters. This is typically achieved by optimizing a criterion, for example, to minimize distances between group members (technically, to minimize withincluster variance), although other objective functions are possible.

Figure $8 \mathrm{~A}$ shows a solution for our working example with five trajectory clusters identified via the bottom-up approach, using Euclidean distance and a basic hierarchical clustering algorithm. Given these clusters, trajectory types can now be understood as the average trajectory within a cluster, emphasized in $8 \mathrm{~A}$. The thus inferred set of types include two almost straight trajectories (representing 34\% and 33\% of all trajectories, respectively), a curved trajectory (15\%), a cluster

\footnotetext{
${ }^{5}$ Note that mt_animate requires installation of imagemagick.
} 
whose average trajectory first goes straight to the non-chosen option before continuing straight to the chosen option (12\%), and one whose trajectories first move towards the chosen and then towards the non-chosen option before eventually reversing course once more (6\%). These clusters and types help illuminate the composition of trajectories in our example data set. They confirm the existence of a substantial number of trajectories that fall between the two dominant types previously discussed (namely the $15 \%$ moderately curved trajectories) and reveal new trajectories not previously visible, such as the $6 \%$ of trajectories that reverse direction twice.

Upon closer inspection, however, the clustering shown in Figure $8 \mathrm{~A}$ does not seem ideal. For instance, the first two clusters are highly similar, suggesting that they could be merged into one. This highlights two fundamental challenges associated with the bottom-up approach. The first challenge is that bottom-up clustering approaches require a set of a priori choices, including, most importantly, the number of clusters to be extracted. Not all such decisions are certain to result in appropriate solutions. For instance, extracting four clusters would likely have produced a more useful result than five in our working example. To address the problem of identifying an appropriate number of clusters one can try to rely on $k$-selection algorithms (Haslbeck \& Wulff, 2020). However, such algorithms also depend on sets of assumptions that may or may not be suitable for the researcher's goals (Hennig, 2015). Ultimately, as clustering is an unsupervised learning problem, there is no way of knowing which assumptions work best without already knowing the solution. The second challenge is that the bottom-up clustering approach is a data-driven approach. This means that the resulting clusters and types will not only depend on predefined assumptions, but also the data. Different datasets will result in different clustering solutions, making comparison between studies difficult if not impossible.

\section{The Top-Down Approach to Trajectory Types}

The top-down approach differs from the bottom-up approach in that it presupposes a set of prototype trajectories. This means that trajectories are no longer grouped based on their similarity to each other. Instead, they are clustered according to their similarity to each of the prototype trajectories. Specifically, trajectories are mapped to the most similar prototype with respect to, for example, Euclidian distance. By predefining prototypes, the top-down approach addresses the two issues associated described above: First, by choosing an appropriate prototype set, it avoids clustering solutions where homogeneous clusters are split in two (as is the case for clusters one and two in Figure 8 A). Second, by defining prototypes a priori, types no longer depend on the dataset under investigation, permitting straightforward statistical comparisons of experimental conditions or other covariates between studies.
The critical ingredient in the top-down approach is the choice of prototype trajectories. In the ideal scenario, the researcher has concrete, a priori hypotheses with respect to the kinds of trajectories that either could occur or would matter in a given study. For instance, in the case of the discrete scenario discussed by Spivey et al. (2005), in which trajectories may either be attracted by the competing option or not, one could specify prototypes that capture these two discrete scenarios. In the absence of a priori hypotheses, prototypes can be borrowed from existing sets, such as the one included in the mousetrap R package, shown in Figure $8 \mathrm{~B}$, that were derived by applying the bottom-up approach to a large number of published data sets (cf. Wulff et al., 2019, Wulff et al., 2021). This set includes five prototypes, representing straight, curved, continuous change of mind ( $c$ CoM), discrete change of mind $(d C o M)$, and discrete double change of mind ( $d C o M 2)$ trajectories. This set may not perfectly capture the kinds of trajectories occurring in all studies, but it likely provides a diverse enough set to produce informative results in most cases. Finally, prototypes can also be generated using the bottom-up approach based on clustering. In this case, however, one should be careful to separate the identification of prototypes from any statistical analysis of the resulting prototype trajectory mappings, for instance by using independent data sets for the bottom-up and top-down analysis steps.

Figure $8 \mathrm{C}$ shows the outcome of mapping the trajectories in our working example to mousetrap's default trajectory prototype set using Euclidian distances. The resulting mapping shares many similarities with the clustering produced by the bottom-up approach, but also some important differences. Where the bottom-up approach had separated straight trajectories into two equally-sized clusters, the top-down approach reliably assigned all into a single large cluster (63\% of all trajectories). Furthermore, where the bottom-up approach lumped together mildly- and strongly-curved trajectories into a single cluster, the top-down approach was able to separate these kinds of trajectories more clearly (curved and $c$ CoM). Overall, the top-down approach seems to have produced a cleaner separation of trajectories into distinct types. It is important to emphasize, however, that no clustering of trajectories is per se better than another, but it can be more useful, depending on the researcher's aims. For instance, whether or not mildly- and strongly-curved trajectories should be treated as one or different types should depend on whether the researcher assigns different meanings to them or not.

\section{Statistical Comparisons of Trajectory Types}

With trajectories mapped to types, statistical comparisons can be carried out in a straightforward fashion. The natural approach to do this is using the frequency distribution of types. Table 1 shows such a distribution for the comparison of typical and atypical trials in our working example. 
A
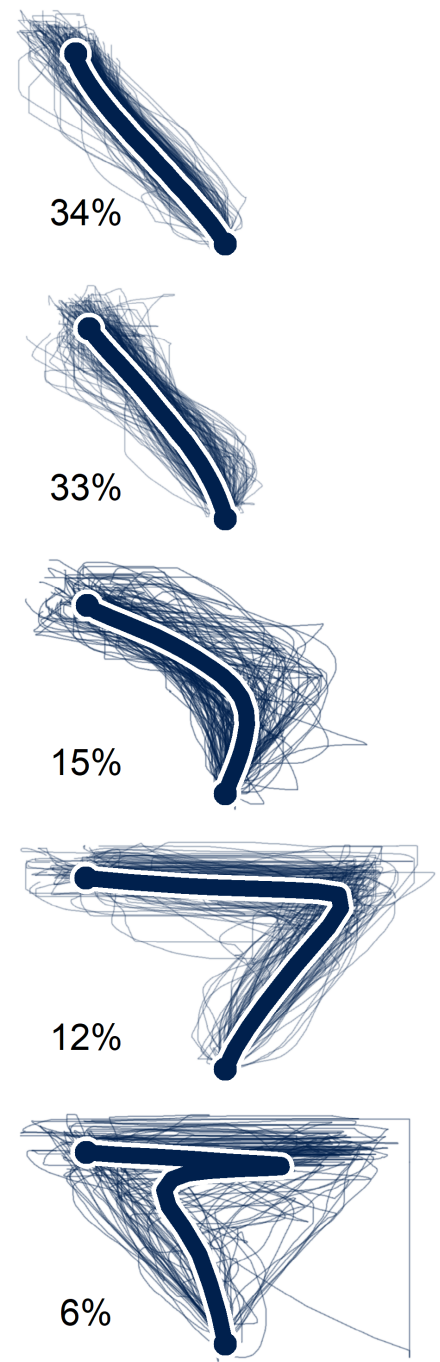

B
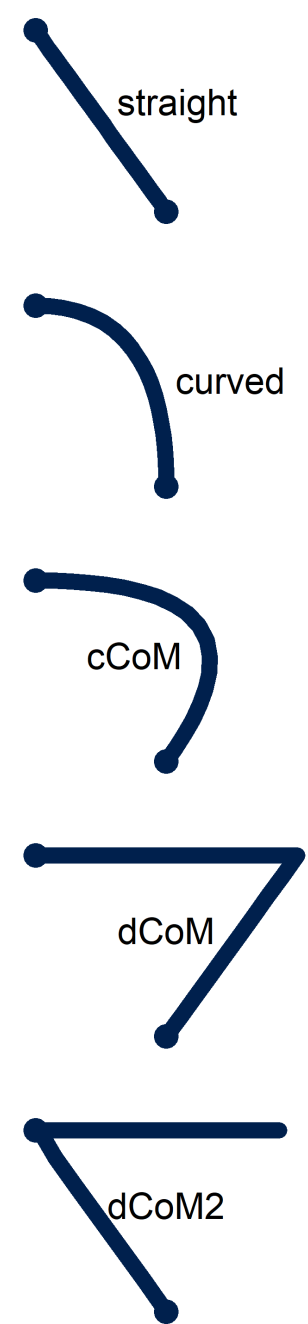

C
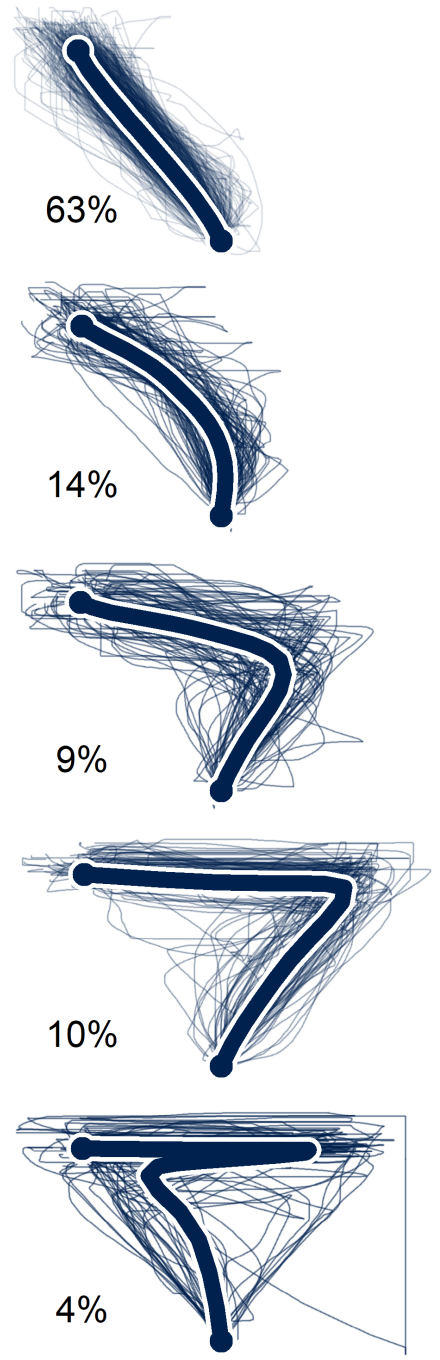

\section{Figure 8}

Clustering movement trajectories. Panel A shows five clusters identified using hierarchical clustering, panel B five of the prototypes presented by Wulff et al. (2019), panel C the clustering based on assigning trajectories to the closest prototype. Thick lines in panel $A$ and $C$ represent the average trajectory.

Consistent with the notion that the manipulation of typicality influences movement trajectories, we observe that trials with atypical animals produced proportionally more $d C o M$ and $d C o M 2$ and fewer straight trajectories, compared to those with typical exemplars. To test these differences against chance, one can use a $\chi^{2}$ test for stochastic independence. This test reveals a significant difference $\left(\chi^{2}(4)=57.97, p<\right.$ $.001)$. Inspection of the residuals, which are also shown in Table 1), reveals that this effect is mostly due to differences in proportions of both change-of-mind trajectory types.

When trajectory types can be ordered along a dimension of interest, researchers can also consider ordinal statistics to compare groups or conditions. For instance, the mousetrap default prototypes could be ordered according to the level of competition that they might reflect: straight trajectories representing the least amount of competition, followed by curved trajectories, continuous and discrete change-ofmind trajectories, and, finally, discrete double change-ofmind trajectories representing the largest amount of competition. This ordering can then be used within ordinal regression models to conduct statistical comparisons. One benefit of this approach is that inferences can be made with reference to the dimension used for ordering, in this case presumed levels of competition. 
Table 1

Type-based Statistical Comparisons of Typical and Atypical Trials: Frequencies and Residuals

\begin{tabular}{lccccc}
\hline Type & Straight & Curved & cCoM & dCoM & dCoM2 \\
\hline \multicolumn{7}{l}{ Frequencies } \\
Typical & 506 & 116 & 54 & 52 & 16 \\
& $(68 \%)$ & $(16 \%)$ & $(7 \%)$ & $(7 \%)$ & $(2 \%)$ \\
Atypical & 165 & 38 & 37 & 56 & 24 \\
& $(52 \%)$ & $(11 \%)$ & $(11 \%)$ & $(18 \%)$ & $(8 \%)$ \\
\hline$\chi^{2}$ & & & & & \\
residu- & & & & & \\
als & & & & & \\
Typical & 1.70 & .80 & -1.21 & -2.71 & -2.26 \\
Atypical & -2.59 & -1.22 & 1.84 & 4.13 & 3.45 \\
\hline
\end{tabular}

\section{Analyzing Trajectory Types using mousetrap}

mousetrap provides implementations of all type-based trajectory analyses described above. Bottom-up clustering of a mousetrap object can be performed using the mt_cluster function. The function will look for length-normalized trajectories, but can, in principle, also work with other trajectory transformations, including time-normalized trajectories. If length-normalized trajectories have not yet been computed, they can be added to the mousetrap object using the mt_length_normalize function, which will represent each trajectory using 20 equidistant points (or as many as are specified using the n_points argument). The mt_cluster function extracts a default number of five clusters using completelinkage, agglomerative hierarchical clustering (Murtagh \& Legendre, 2014). However, all relevant aspects of the clustering procedure, including the number of clusters (n_cluster argument) and the clustering algorithm (method argument), can be adjusted. The result of mt_cluster is stored in a new data frame element within the mousetrap object called clustering. Top-down prototype mapping can be performed using the mt_map function. By default, the function maps trajectories to the mousetrap standard prototype set (see Figure 8) based on the Euclidean distance; however, both the prototype set and the distance function can be flexibly adjusted. The result of mt_map is stored in a new data frame element in the mousetrap object called prototyping.

The code below shows how to apply these approaches to our working example. The first chunk uses mt_length_normalize to length-normalize the trajectories, which provides the basis for the steps that follow. The second chunk uses mt_cluster to extract the default of five clusters using the bottom-up approach. mt_plot is used with use2 = "clustering" and facet_col = "cluster" to produce a visualization of the clustering similar to Figure 8 A (but with the clusters spread out over columns instead of rows). The third chunk uses mt_map to perform topdown clustering using mousetrap's standard prototype set and then similarly visualizes the resulting clustering using mt_plot. Building on the third chunk, the fourth chunk produces a table of the prototype mapping for typical and atypical trials and compares the respective frequencies using the chisq.test. The final chunk uses the clmm function of the ordinal package (Christensen, 2019) to perform the same comparison using ordinal statistics, with mousetrap's prototypes ordered as suggested in the previous section.

\# 1) Length-normalize trajectories ------

mt_data <- mt_length_normalize(mt_data)

\section{\# 2) Bottom-up clustering ------}

\# Perform bottom-up clustering approach mt_data <- mt_cluster(mt_data)

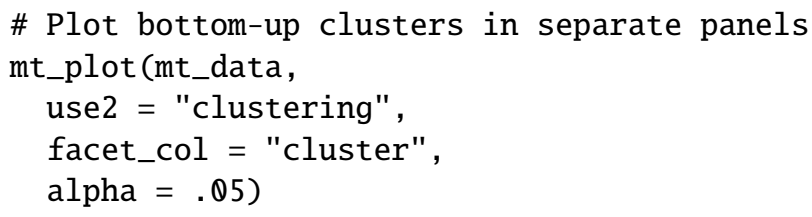




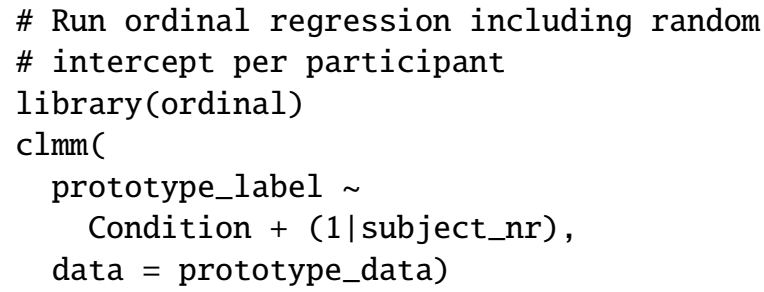

\section{Temporal Evolution of the Trajectory}

The alternative route towards analyzing the dynamics of cognitive processes using movement tracking focuses on the temporal development of key trajectory features. Typically, the main goal behind this approach is to reveal when and how strongly movements commit towards the chosen option. In the following sections, we will discuss the temporal evaluation approach to movement tracking and illustrate it using our working example. Note that some of the assumptions underlying this approach are not met by our working example. We will discuss this point after we have presented the approach.

The simplest approach to analyzing the temporal evolution consists in analyzing aggregate trajectory features across time. Figure 9 illustrates this for the position on the horizontal axis and movement angle using the data of our working example. Specifically, panel $9 \mathrm{~A}$ shows the position on the horizontal axis as a function of the trajectories' time steps; that is, proportions of the trial duration as determined using time-normalization (see section Resampling Trajectories), with the foreground showing aggregate trajectories, calculated from the typical (yellow) and atypical (blue) raw trajectories displayed in the background. The analysis reveals distinct temporal developments for the types of trials: The aggregate trajectory for typical trials turns towards the eventually chosen option, located at an x position of -625 pixels, sooner than the aggregate trajectory for atypical trials, suggesting an earlier choice commitment in typical as compared to atypical trials. The difference between the aggregate trajectories is significan ${ }^{6}$ between time steps 51 and 95, as indicated by the line of asterisks at the top. Panel $9 \mathrm{~B}$ ) shows an analogous analysis for the movement angle relative to the vertical axis, which is 0 when the movement is directed straight upwards, $\pi$ if the movement is directed $90^{\circ}$ to the left, the side of the chosen option, and $-\pi$ if the movement is directed to the right. In this analysis, two larger regions of consecutive significant differences emerge. First, typical trials deviate significantly from atypical trials between time steps 47 and 71 in the direction of positive angles, indicating a movement towards the chosen option. Then, between time steps 78 and 101 the pattern turns around with atypical trials now deviating in the direction of positive angles, indicating that they are only now turning towards the chosen option, while many typical trials have already reached their final horizontal position in the response box.
In principle, the development of any trajectory feature can be analyzed in this fashion. However, with few exceptions, studies have focused on the horizontal axis and angle relative to the vertical axis, two features usually interpreted as signals of the relative commitment between options (see Stillman et al., 2018b). One exception is provided by Koop and Johnson (2013), who studied the temporal development of velocity and acceleration, with the similar aim of drawing inferences about the strength and time course of choice commitment.

\section{Modeling the Temporal Dynamics of Trajectories}

A more sophisticated approach to analyzing the temporal evolution uses statistical models to test the effect of one or more independent variables or covariates across time. Commonly, this is done in a step-by-step fashion, such that each time step is analyzed separately using general linear models (Dale \& Duran, 2011; Scherbaum et al., 2010). In the case of a single categorical predictor, this approach is equivalent to the analysis presented in Figure 9, which revealed that movements in atypical trials tended to commit to the chosen option later than typical ones. Although identifying the time point at which a single factor acts on the trajectories in a study can be useful, the real value of temporal analyses only emerges once multiple predictors are considered in a model. For instance, the analysis of our working example could have additionally considered word frequency as another driver of the categorization process (e.g., Brysbaert et al., 2018, Forster, 2004). Including both typicality and word frequency in a joint model produces two sets of regression weights across time. These can then be used to infer the relative strength and order in which the predictors influence the movement and, by extension, the cognitive proces: 77 Studies have used this approach, for example, to show that the tastiness of food is processed earlier than its healthiness (Sullivan et al., 2015), or that values are processed earlier than delays in an intertemporal choice task (Dshemuchadse et al., 2013). Most such analyses have used movement angle as the dependent variable, but, in principle, they can also be carried out using movement position or other features of the trajectory.

\footnotetext{
${ }^{6}$ Tested using mixed models that were run per time step and included a fixed effect for the condition and random intercepts per participant. Note that previous analyses have sometimes used a different approach: They first averaged the measure of interest (e.g., the $\mathrm{x}$ position or movement angle) per participant, time step and condition, and then performed paired t-tests between conditions per time step. However, this approach does not appropriately take into account differing numbers of trials between participants. These occur, for example, for movement angles when there is no movement between time steps, which frequently occurs in early stages of the trial.

${ }^{7}$ It has been proposed that Gaussian mixtures could be used to additionally infer the duration, peak strength, and peak duration of the effect of different predictors on the movement trajectory (Scherbaum \& Dshemuchadse, 2019).
} 

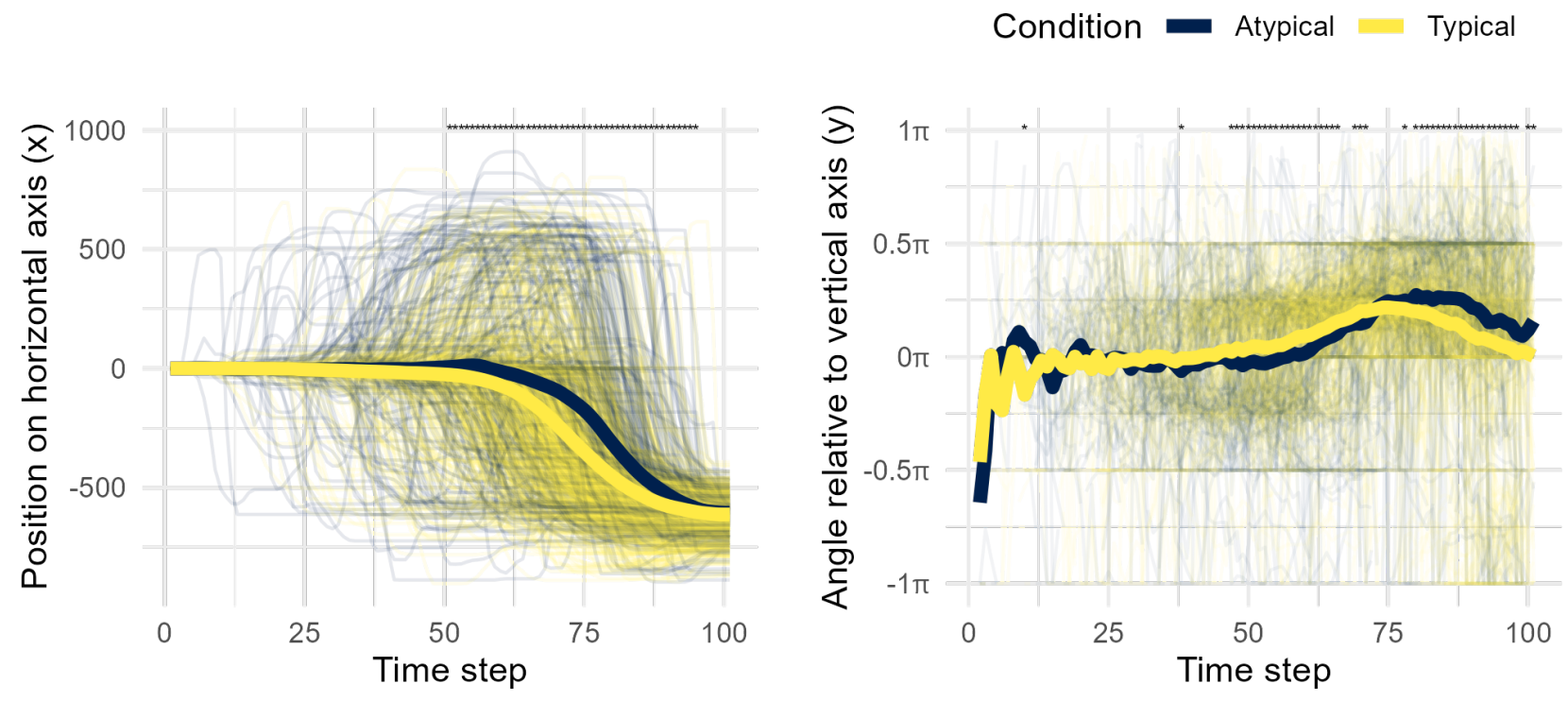

\section{Figure 9}

Temporal dynamics across time steps. Panels $A$ and $B$ show the effect of typicality on the trajectories' $x$-axis positions (A) and angles relative to the vertical axis $(B)$ across 101 time steps. Aggregate trajectories of typical (yellow) and atypical (blue) trials in the foreground are the arithmetic mean of the respective individual trajectories shown in the background. The line of asterisks at the top of each panel indicates regions where the average lines differ significantly from each other according to separate linear mixed models per time step and $\alpha=.05$. See text for details on statistical analyses.

When using the step-by-step regression approach it is important to be aware of its underlying assumptions. First, as discussed in a previous section, temporal analyses including especially the step-by-step regression approach assume trajectory homogeneity. This assumption is necessary for aggregate trajectory features to be representative of the distribution from which they are calculated, and may also be required by the statistical models recruited. Note that this assumption is not met by our working example, implying that the presented analysis should be interpreted with caution. Second, modeling the time steps separately implicitly assumes independence between time steps, although clearly this is not the case. This introduces challenges for proper statistical inference due to multiple testing. It has been suggested that this issue can be addressed by considering only a certain minimum number of consecutive significant results (e.g., Dale et al., 2007). However, ultimately, the only appropriate approach to deal with multiple testing is a joint statistical mode ${ }^{8}$ Third, temporal analyses implicitly assume that the predictors are uncorrelated with absolute trial duration. This assumption arises from the use of time-normalization, where trajectories of different duration are resampled to the same number of points. As a result, points at the same relative time may be at very different positions in absolute time. This can be problematic when the goal is to derive conclu- sions with respect to absolute time. Consider, for instance, the difference between the aggregate trajectories of typical and atypical trials 9A. To be able to interpret this difference as an early commitment for typical as compared to atypical trials with respect to absolute time, typical and atypical trials must be roughly equal in their average response time. When they are not, it is at least conceivable that the true order of commitments is reversed, namely when typical trials take considerably longer in absolute time than atypical ones.

To ensure that the results of temporal analyses, andspecifically - the step-by-step regression approach, are not confounded by differences in absolute time, researchers have, at least, two options. First, they can inspect the distribution of absolute response times as a function of the independent variables. When relationships exist, it is important to assess whether or not they are in conflict with the results obtained in the temporal analysis. For instance, Figure 10A shows the distribution of response times in our working example as a function of typicality. Clearly, a relationship exists between response time and typicality, in that typical trials $\left(\right.$ median $\left._{R T}=1519.5\right)$ are, on average, much faster than atypical ones $\left(\right.$ median $\left._{R T}=2032.5\right)$. However, this difference, if anything, emphasizes the claim of earlier

\footnotetext{
${ }^{8}$ See Cox et al. 2012) for one proposal in this direction
} 
commitment in typical trials as compared to atypical trials. The second option is to rerun the temporal analysis for different strata of the response time distribution in which response times are comparable, to assess robustness. The middle panels of Figure 10 show this for trials with response times between 0 and 1,499ms (Figure $10 \mathrm{~B} ; 39 \%$ of trials), trials between 1,500 and 2,500ms (Figure $10 \mathrm{C} ; 44 \%$ ), and between 2,500 and 5,000ms (Figure 10 C; 13\%). The panels show an earlier commitment for typical as compared to atypical trials in all three strata, suggesting once more that the qualitative results of the analysis also hold in absolute time. However, the magnitude and timing of effects seem to vary somewhat across the strata. Specifically, for small response times, the effect is smallest and seems to emerge at about $500 \mathrm{~ms}$, whereas for medium response times, the effect is considerably larger and seems to emerge at about twice the absolute time, namely about $1,000 \mathrm{~ms}$, and for large response times the effects seems to largest, emerging again at about $1,000 \mathrm{~ms}$. These patterns are likely the result of types uncovered in the previous sections. Figure $10 \mathrm{E}$ illustrates the distribution of response times as a function of trajectory types, which shows that small absolute response time trajectories are overwhelmingly of type straight and curved, whereas the $c C o M, d C o M$, and $d C o M 2$ types only enter with larger response times. This illustrates again, that the effect of typicality hinges on change-of-mind trajectory types.

\section{Analyzing the Temporal Evolution using mousetrap}

mousetrap provides functions to calculate and aggregate relevant trajectory features across time. The mt_angles function calculates angles between two-point segments and the vertical axis, as well as angles at the center of three-point segments. The $\mathrm{mt}$-derivatives function calculates distances, velocities and acceleration values. Features are by default calculated for and added to the standard trajectory element. However, via the use argument they can also be added to other trajectory elements available in the mousetrap object. To aggregate trajectory features across time, the mt_aggregate function can be used. To visualize trajectory features across time, mt_plot and mt_plot_aggregate can be used to plot the temporal development for individual and aggregated trials per condition respectively.

The code below shows how to compute and visualize trajectory features across time. The first chunk uses mt_time_normalize to add time-normalized trajectories to the mousetrap object, which will be used in the steps that follow. The second chunk uses mt_plot and mt_plot_aggregate to plot the individual and aggregated $\mathrm{X}$-position across time within the same figure using ggplot2 addition. Setting use = "tn_trajectories" ensures the plot is based on the time-normalized trajectories, whereas setting $\mathrm{x}=$ "step" and $\mathrm{y}=$ "xpos" ensures that time steps and $\mathrm{x}$-position are placed on the $\mathrm{x}$ and $\mathrm{y}$ dimensions of the plot. Setting color $=$ "Condition" results in separate colors for trajectories belonging to typical and atypical trials. Note that the addition of individual and aggregate visualizations is enabled by the setting return_type = "geom" in mt_plot_aggregate. The third chunk uses mt_angles to compute angles for the time-normalized trajectories and then visualizes the angles in a fashion analogous to the approach used for x-positions 9 .

\# 1) Time-normalize trajectories ------

mt_data <- mt_time_normalize(mt_data)

\# 2) Plot $\mathrm{x}$-positions across time -----

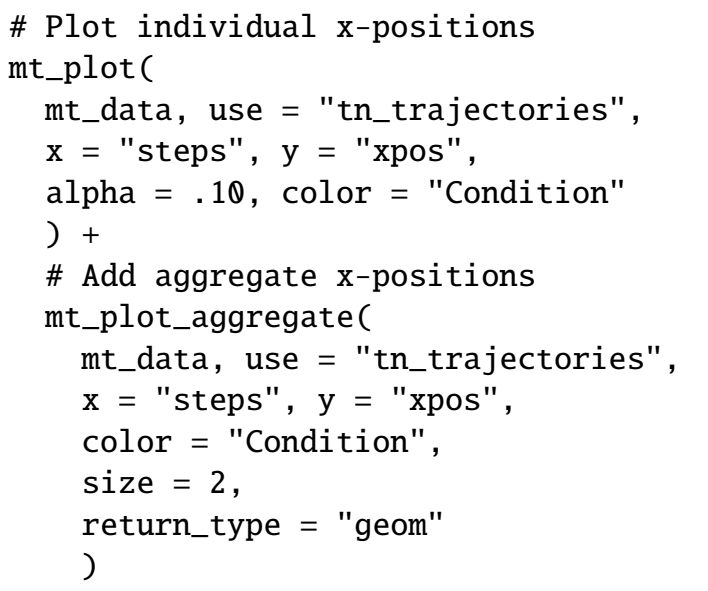

\# 3) Plot angles across time -----

\# Calculate angle relative to vertical axis \# for time-normalized trajectories

mt_data <- mt_angles ( mt_data, use = "tn_trajectories")

\# Plot individual angles

mt_plot ( mt_data, use = "tn_trajectories", $\mathrm{x}=$ "steps", $\mathrm{y}=$ "angle_v", alpha $=.10$, color $=$ "Condition" ) +

\# Add aggregate angles mt_plot_aggregate( mt_data, use = "tn_trajectories", $\mathrm{x}=$ "steps", $\mathrm{y}=$ "angle_v", color = "Condition", size $=2$, return_type = "geom",

\footnotetext{
${ }^{9}$ Note that, in this case, a custom aggregation function is employed to deal with the missing values arising from a lack of movement between some time stamps.
} 
A
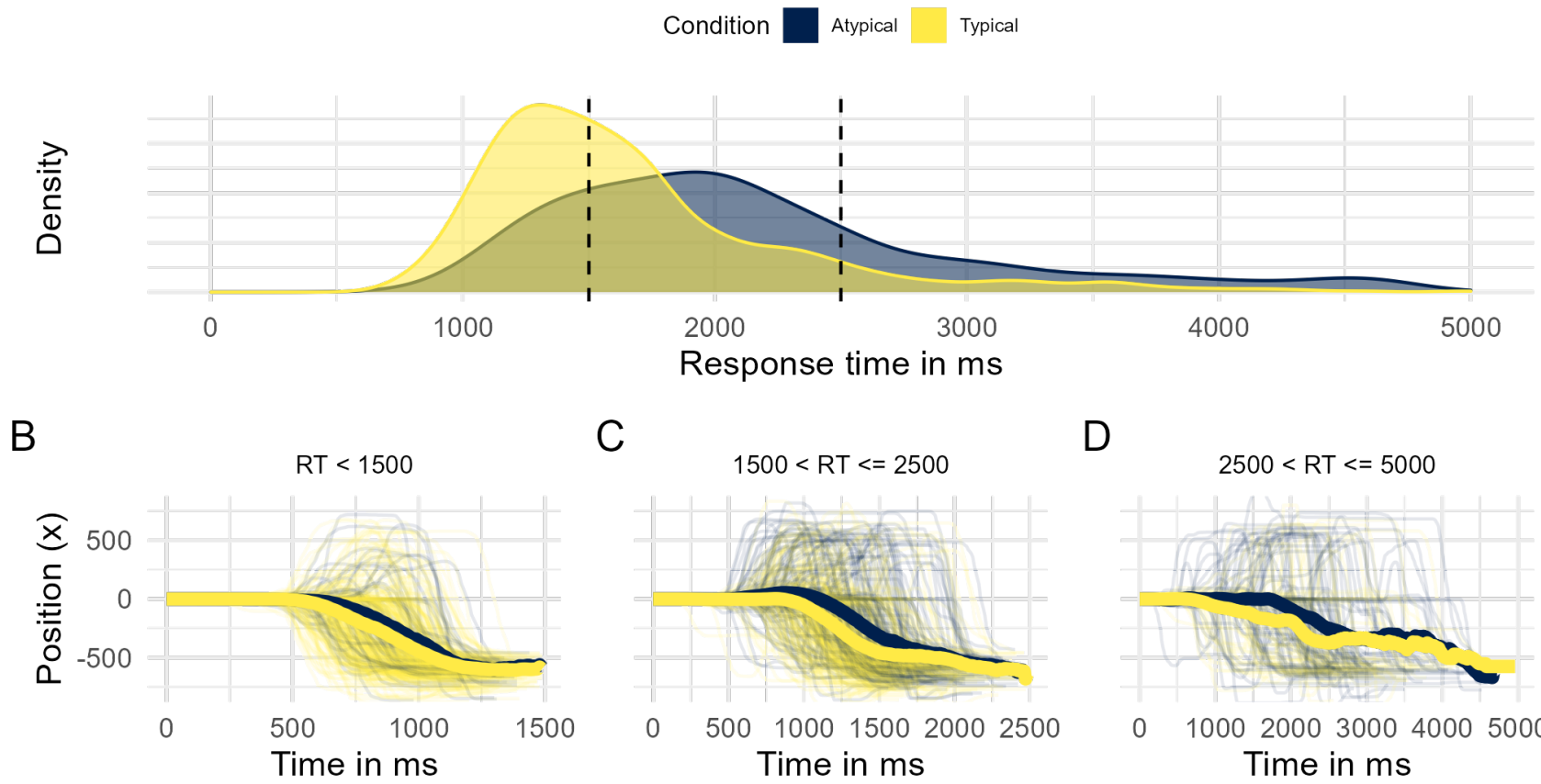

C
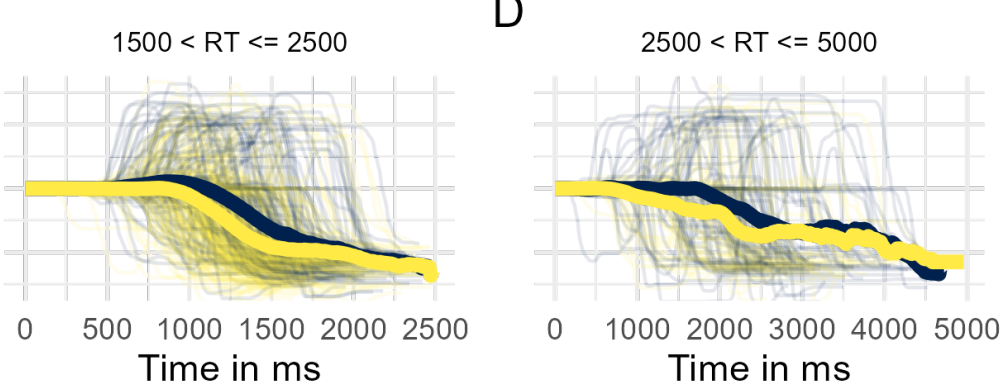

$E$ Trajectory type $\square$ straight $\square$ curved $\square$ cCoM $\square$ dCoM $\square$ dCoM2

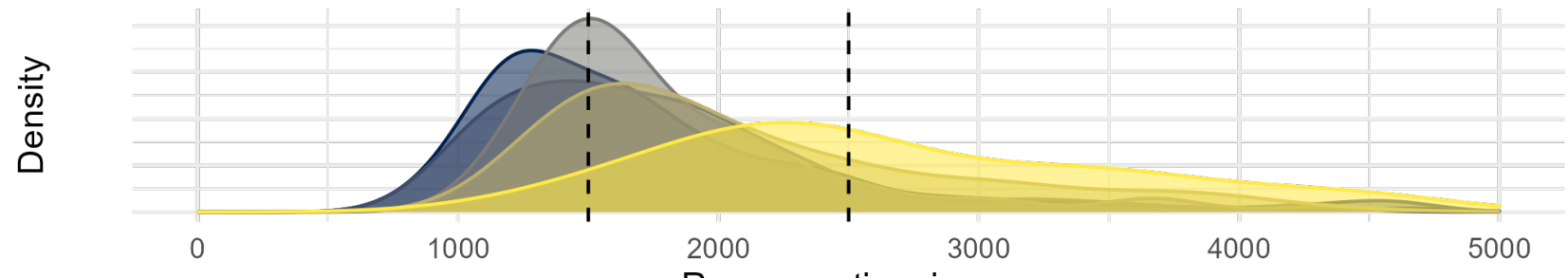

Figure 10

Temporal dynamics in absolute response time. Panel A shows the distribution of absolute response times of typical (yellow) and atypical (blue) trials in ms. Vertical dashed lines indicate the 1,500ms and 2,500ms cutoffs used for panels B to D. Panels $B$ to $D$ show the effect of typicality on the trajectories' $x$-axis positions across absolute time for trials with absolute response of between 0 and 1,500ms (B), 1,500 to 2,500ms (C), and 2,500 to 5,000ms (D), respectively. Panel E shows the distribution of response times as a function of trajectory type (see Figure 8). In all figures, trajectories with response times $>5,000$ ms were excluded (33 trajectories).

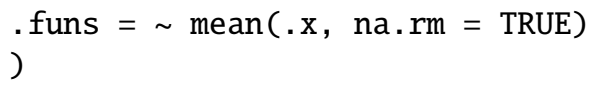

\section{Theoretical and Practical Considerations}

Thus far, our article has mostly focused on how an existing movement tracking data set can be analyzed in different ways to learn more about the underlying cognitive processes. In this section, we discuss two general issues that should be considered in the design stage of a movement tracking study. First, we will discuss different conceptions of the mapping between movement trajectories. Second, we will discuss the importance of study design choices.

\section{The Mapping Between Cognitive Process and Movement}

The traditional perspective on movement tracking is that of a continuous process of evidence accumulation that con- 

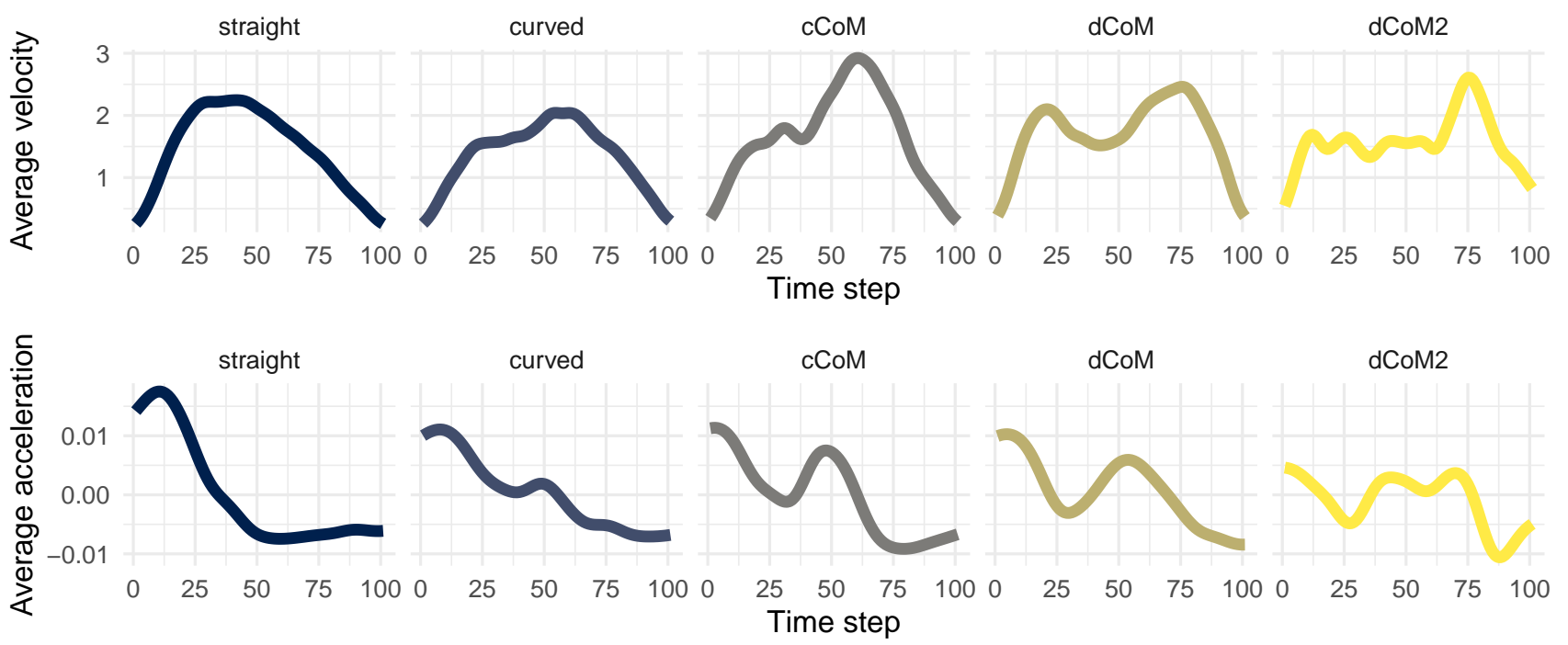

\section{Figure 11}

Velocity and acceleration profiles of trajectory types. The two panels show, respectively, the average velocity and acceleration in pixels per time step of trajectories assigned to our five prototypes. To better align the temporal development of trajectories and reduce noise, initiation times and idle times immediately preceding choice were cropped from the raw trajectories, before velocity and acceleration were computed. Afterwards velocity and acceleration were time-normalized and smoothed across time steps using a Gaussian filter.

tinuously translates into movement. This view is clearly embodied by existing computational accounts of movement tracking processes, including attractor models (Frisch et al., 2015, Scherbaum et al., 2016; Spivey et al., 2005, Zgonnikov et al., 2017) and drift-diffusion models (Calluso et al., 2018; Wong et al., 2015). According to these models, a latent preference for the available option evolves until either a stable (attractor models) or an extreme enough (diffusion models) point of preference for one of the options is reached. This ongoing evolution of preference is thought to translate immediately and continuously into features of the movement, such as its current angle (wong_motor_nodate) or location (Spivey et al., 2005). However, while accumulator and diffusion models today are widely accepted as plausible models of human decision processes (Busemeyer et al., 2019; Gold \& Shadlen, 2007), the literature has not yet settled whether or not accumulation or other kinds of processes translate directly into movements .

The alternative perspective is that cognitive processes and movements are linked in a more intermittent fashion (Friedman et al., 2013). Support for this position comes from research on motor control in basic reaching tasks. According to the predominant view in this field, reaching consists of multiple sub-movements, usually involving an initial pre-planned, fast, ballistic movement used to get the limb into the target area and one or more smaller and slower corrective movements (Battaglia-Mayer et al., 2014, Elliott et al., 2010).
Similar proposals have been made in research on humancomputer interaction (Martın et al., 2021). It stands to reason that if these basic reaching movements are controlled intermittently, then maybe this also holds for reaching movements that are the product of higher-order cognitive processes.

Recent movement tracking studies have reported evidence that clearly favors the intermittent view for at least some trajectories (Freeman, 2014; Friedman et al., 2013, Kieslich et al., 2020, Tomlinson Jr et al., 2013, Wulff et al., 2019). Specifically, these studies have observed trajectories involving abrupt directional changes. The analysis of our working example mirrors these findings. First, the type-based analyses presented above have revealed several different types of trajectories, including substantial proportions of discrete change-of-mind trajectory types ( $d C o M$ and $d C o M 2$ ), which likely are the product of large independent sub-movements. Second, further analysis of the remaining trajectories focusing on velocity and acceleration suggests that even these may be the result of intermittent control. Figure $11 \|^{10}$ shows that there are distinct dips in the velocity and acceleration profiles at consistent relative positions across all trajectory types, except straight trajectories. These dips reflect pauses in the movement, possibly as a result of the cognitive pro-

\footnotetext{
${ }^{10}$ For better alignment, any initiation and idle periods before choice have been removed from the underlying trajectories. Also note that, due to the reliance on time-normalized trajectories, dips may occur at different positions in absolute time.
} 
cess controlling and re-orienting the movement at the these points. This suggests that all trajectories, including curved and $c$ CoM, could possibly have been the the result of a small number of intermittently controlled sub-movements.

If the link between cognitive process and movement were an intermittent one, it would have implications for the interpretation of movement trajectories. Consider, for instance, the $d C o M$ trajectory type. Under the traditional, continuouslink view, such a trajectory would have to be interpreted as an effectively discrete decision process that first strongly prefers the alternative option until it almost reaches the threshold for a decision, before reversing completely to a strong preference for the eventually chosen option. Under an intermittentlink view, however, preferences could evolve gradually, but we would not be able to know when and how, aside from obvious changes in direction or speed. This means that, under an intermittent-link view, one can only be confident about the discrete sequence of preference shifts, but not the level of preference nor its exact temporal evolution. This means that conceivably curved, $c C o M$, and $d C o M$ trajectories might result from the same underlying cognitive process.

Finally, one must also consider that the cognitive process itself could be discrete in nature. Many models of cognition are inherently discrete by being based on, for instance, serial memory search (Raaijmakers \& Shiffrin, 1981; Wulff et al., 2020, e.g., ), serial information acquisition (Krajbich et al., 2012; Pachur et al., 2018, Wulff et al., 2018, e.g., ), or lexicographic decision rules (Hertwig et al., 2019; Johnson et al., 2008, Şimşek, 2020, e.g., ). Such discrete processes would produce discrete movements, even if the link between process and movement were a continuous one.

Future research will have to clarify the nature of cognitive processes and their link to movement trajectories. One possibility that this research needs to consider is that the nature of these two theoretical components, process and link, are a function of the particular study subject and design. In the next section, we will review findings that make suggestions along these lines.

\section{The Role of Study Design}

Designing movement tracking studies involves several key choices, including, importantly, selecting and setting up a recording device, and selecting the starting procedure, layout, and response mode used in trials of the study. Past work has shown that these choices play an important role in shaping the recorded movement trajectories. Specifically, they have been found to impact, among other characteristics, the magnitude of experimental effects, such as, for instance, the effect of typicality on trajectory curvature in our working example, and the level of homogeneity of movement trajectories (Blinch et al., 2018; Burk et al., 2014; Grage et al., 2019, Kieslich et al., 2020, Scherbaum \& Kieslich, 2018, Schoemann et al., 2019, Schoemann et al., 2021; Wirth et al.,
2020).

Table 2 gives an overview of movement tracking design aspects and associated empirical findings. To highlight a few results, larger rather than smaller distances between response options and a click rather than a hover response mode have been linked to larger experimental effects. On the other hand, the use of robotic handles and camera-based rather than other tracking devices, dynamic rather than static starting procedures, and touch rather than click response modes have been linked to larger homogeneity. See Figure 12 for an illustration of the effect of design on trajectory homogeneity based on the data by Kieslich et al. (2020). Notably, the magnitude of experimental effects and trajectory homogeneity have been found to be inversely related, with large effects being associated with lower homogeneity and vice versa. This has, for instance, been the case for small versus large distances between response options (Burk et al., 2014, Wirth et al., 2020), click versus hover response modes (Grage et al., 2019, Kieslich et al., 2020), and possibly timed and dynamic versus static starting procedures, though here the case may be more complex (see Kieslich et al., 2020).

The strong role of study design could have important implications for designing and interpreting movement trajectory studies. The strong effect on homogeneity suggest that design choices could affect the link between cognitive process and movement trajectory, with designs associated with homogeneous trajectories possibly triggering a more continuous link and designs associated with heterogeneous trajectories possibly triggering a more intermittent link. The negative relationship between the magnitude of experimental effects and trajectory homogeneity further suggests that a potentially continuous link does not necessarily result in larger signals of the underlying process. More than anything else, this would underpin that the usefulness of movement tracking is not dependent on subscribing to the traditional perspective of a continuous link between cognitive process and movement. However, it is important to point out that the number of studies investigating the role of study design is still small and and many design aspects have not yet been systematically investigated either by themselves or in combination with other devices. More research will be needed to fully elucidate the role of study design for movement tracking and which setups are most suited for different purposes.

\section{Recommendations}

Movement tracking is a relatively young methodology, with both practical and theoretical questions yet to be settled. Even so, the literature has made considerable progress in establishing movement tracking as a useful tool for studying cognitive processes. In this final section, we summarize several key takeaways and derive concrete recommendations that should help researchers make the best possible use of movement tracking. 
Table 2

A Summary of Design Aspects of Movement Tracking Studies and Findings Concerning their Role in Shaping Movement Trajectories.

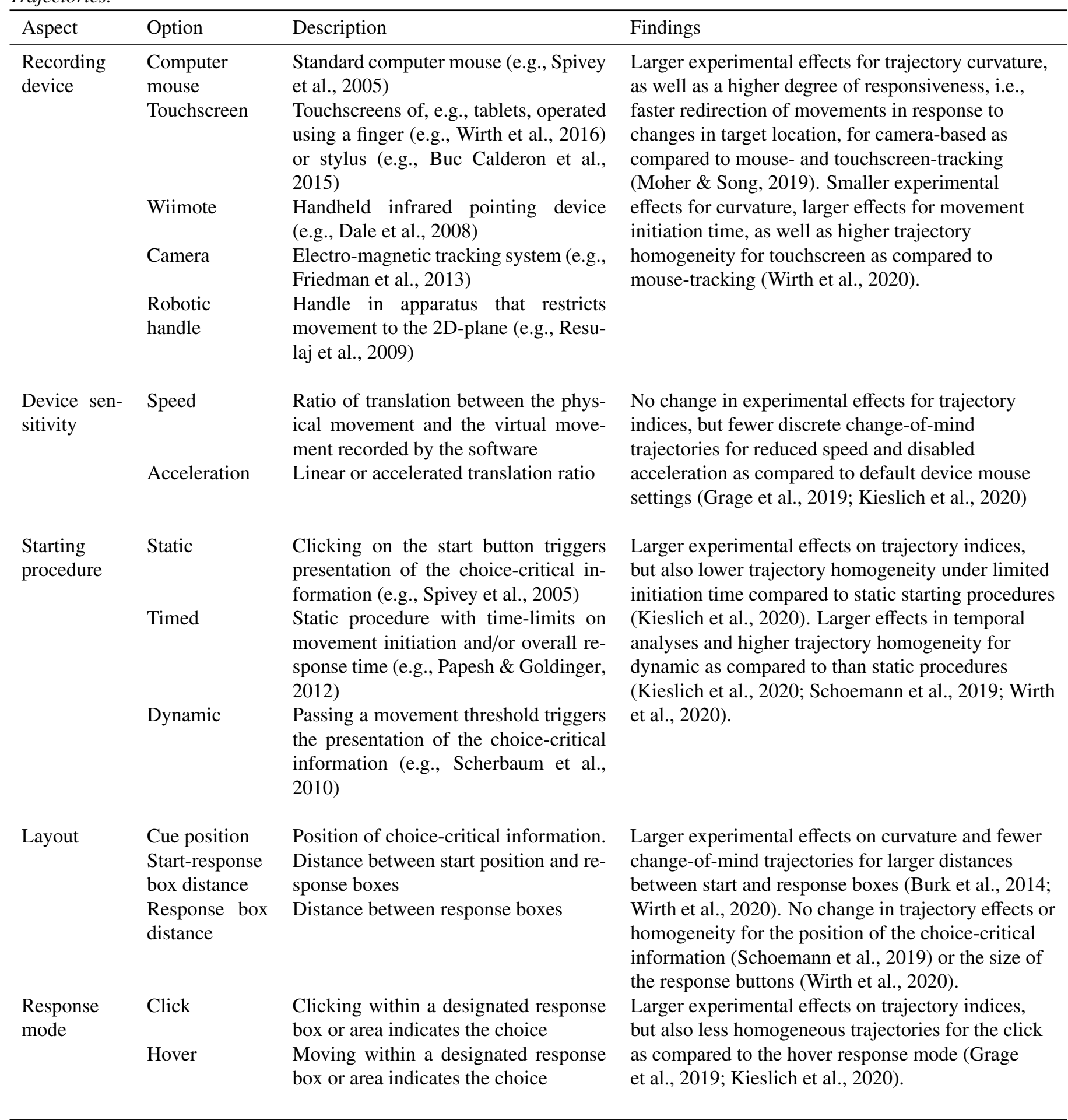


A
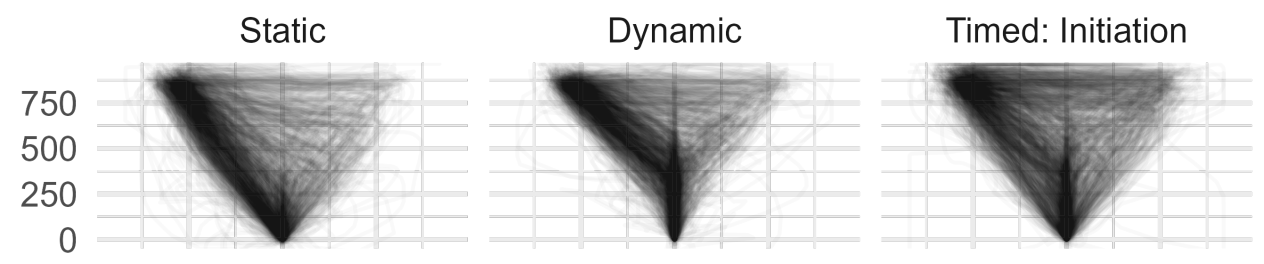

Timed: Response

B

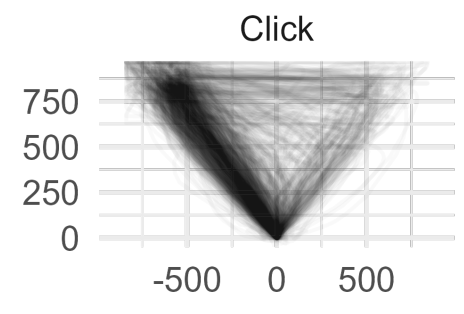

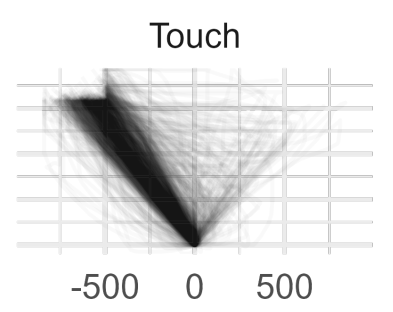

C

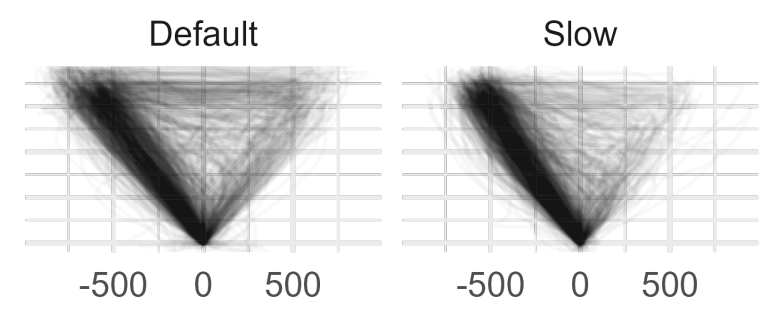

Figure 12

Impact of trial design on trajectory homogeneity. The figure displays trajectories obtained under the eight different study designs examined in Kieslich et al. (2020). Panel A shows trajectories for four different implementations of the starting procedure. Panel B compares the click and the touch response mode. Panel C focuses on two common device-sensitivity settings, default settings under Windows (medium speed and acceleration enabled), and slow settings (reduced speed and acceleration disabled).

\section{Implementation}

Study setup. The experimental setup of movement tracking studies impacts the magnitude of experimental effects and trajectory homogeneity, which have been found to be inversely related. Larger experimental effects but also fewer homogeneous trajectories are associated with static versus dynamic starting procedures, click versus hover response modes, and wide versus narrow option layouts. The setup of a movement tracking study should ultimately be chosen with the theoretical background and the planned analyses in mind. Higher temporal resolution of the cognitive process in the movement trajectory might possibly be achieved by designs associated with homogeneous trajectories. Conversely, larger effects and likely richer behavioral outcomes, including one or multiple changes of minds, might be achieved by designs associated with heterogeneous trajectories.

\section{Processing}

Filtering. Movement tracking produces data that can benefit from filtering. Theoretically, filtering seeks to remove trajectories that do not reflect the intended cognitive processes, for example, decision errors or lack of attention. Trajectory filtering should be kept to a minimum and avoided wherever possible; in any case, trajectory cleaning must be explicitly documented.

Spatial transformations. Mapping trajectories to a single side as well as starting point alignment are common and uncontroversial steps during trajectory processing. Transformations that stretch or compress trajectories or change their original coordinate system should be avoided, unless the aim is to render trajectories comparable across study setups.

Resampling. Trajectory resampling is a common, but not always necessary nor inconsequential processing step. Resampling choices should be made with the analysis in mind. Time-normalization facilitates temporal analyses, for example, the step-by-step trajectory regression. Lengthnormalization is suitable for type-based analyses, for example, clustering. Wherever possible researchers should test for unexpected effects of resampling by carrying out the analysis using the raw data. mousetrap facilitates this by storing resampled trajectories as new elements, retaining the raw data.

\section{Analysis}

Analysis pathway. There are three main analysis pathways, focusing on trajectory indices, the trajectories' temporal evolution, and trajectory types. The choice between these should be motivated by the theoretical questions and should match the distribution of trajectories at hand. Analyses of trajectory indices and, in particular, temporal analyses are based on the assumption of homogeneous trajectories. Typebased analyses are best suited for heterogeneous trajectories. Trajectory homogeneity should always be assessed, ideally using visualizations of individual, unprocessed trajectories. 
Trajectory indices. Trajectory indices characterize trajectories along three main dimensions: curvature, complexity, and time. Curvature indices characterize how strongly a trajectory is bent towards the non-chosen option, and can be distinguished into point-based and integrative variants. Correlations among trajectory indices are usually extremely high $(r \sim 9)$, implying that the choice of curvature index might be motivated by ease of interpretation, which favors $M A D$ and $A D$. Complexity indices characterize a trajectory's level of disorder. Flips, Reversals, and Sampleentropy capture the number, locations, and unpredictability of directional changes. Correlations among complexity indices are high $(r \sim .5)$, yet small enough to capture unique information. Temporal indices characterize the temporal aspects of the trajectory by decomposing the total response time $R T$. Initiationtime measures the duration until movement initiation, whereas Idletime and Movementtime measure the still and active parts after initiation. Idletime and Movementtime are highly similar to $R T(r \sim .7)$, but may capture unique nuances. Initiationtime, however, is largely uncorrelated and could capture unique psychological aspects. All three types of trajectory indices have been theoretically linked to and are sensitive to manipulations of response competition. Complexity indices have additionally been linked to decision wavering, whereas Initiationtime has been linked to pre-movement deliberation. Correlations between index types suggest a moderate degree of redundancy.

Trajectory types. Type-based analyses can be subdivided into a bottom-up and top-down approach and typically recruit length-normalized trajectories. The bottom-up approach uses cluster analysis to extract clusters of trajectories from a data set. It is a useful exploratory tool for characterizing the kinds and homogeneity of trajectories, and can be used as a precursor for the top-down approach. The top-down approach maps trajectories onto a predefined set of prototypes. Prototypes are best derived from theory, or, if they are derived bottom-up, learned from a separate data set. The mousetrap prototype set is a useful default, but may not fit every study setup. Type-based analyses should be chosen when trajectories are heterogeneous. They can reveal which kinds of trajectories are most sensitive to experimental manipulations or covariates, and might identify distinct cognitive processes, such as changes-of-mind. Statistical analyses should be carried out on a nominal or, at most, an ordinal scale level.

Trajectory evolution. Temporal analyses characterize the evolution of trajectory features, such as position, angle, or velocity, across time steps. Temporal analyses are usually based on time-normalized trajectories, but they can also be carried out using raw trajectory data. Temporal analyses can reveal the timing, order, and possibly the strength at which decision-relevant pieces of information affect the movement. When using time-normalized trajectories, one should be very careful in making statements on the absolute timing of events. Temporal analyses should be avoided when trajectories are highly heterogeneous.

\section{Conclusions}

Movement tracking is a maturing process tracking technology that is finding wide adoption across the cognitive sciences for its ability to elucidate the temporal development of preference formation. We hope that the mousetrap R package and this tutorial will help researchers apply movement tracking to generate novel empirical insights into their own research questions and advance movement tracking as a process tracing technology.

\section{References}

Abney, D. H., McBride, D. M., Conte, A. M., \& Vinson, D. W. (2015). Response dynamics in prospective memory. Psychonomic Bulletin E Review, 22(4), 1020-1028.

Bates, D., Mächler, M., Bolker, B., \& Walker, S. (2015). Fitting linear mixed-effects models using lme4. Journal of Statistical Software, 67(1), 1-48. https://doi. org/10.18637/jss.v067.i01

Battaglia-Mayer, A., Buiatti, T., Caminiti, R., Ferraina, S., Lacquaniti, F., \& Shallice, T. (2014). Correction and suppression of reaching movements in the cerebral cortex: Physiological and neuropsychological aspects. Neuroscience $\mathcal{E}$ Biobehavioral Reviews, 42, 232-251.

Blazej, L. J., \& Cohen-Goldberg, A. M. (2015). Can we hear morphological complexity before words are complex? Journal of Experimental Psychology: Human Perception and Performance, 41(1), 50-68.

Blinch, J., Kim, Y., \& Chua, R. (2018). Trajectory analysis of discrete goal-directed pointing movements: How many trials are needed for reliable data? Behavior Research Methods, 50(5), 2162-2172. https://doi. org/10.3758/s13428-017-0983-6

Bogacz, R., Brown, E., Moehlis, J., Holmes, P., \& Cohen, J. D. (2006). The physics of optimal decision making: A formal analysis of models of performance in two-alternative forced-choice tasks. Psychological review, 113(4), 700.

Brysbaert, M., Mandera, P., \& Keuleers, E. (2018). The word frequency effect in word processing: An updated review. Current Directions in Psychological Science, 27(1), 45-50.

Buc Calderon, C., Verguts, T., \& Gevers, W. (2015). Losing the boundary: Cognition biases action well after action selection. Journal of Experimental Psychology: General, 144(4), 737-743. https://doi.org/10.1037/ xge0000087 
Burk, D., Ingram, J. N., Franklin, D. W., Shadlen, M. N., \& Wolpert, D. M. (2014). Motor effort alters changes of mind in sensorimotor decision making. PLOS ONE, 9(3), e92681. https://doi.org/10.1371/journal. pone.0092681

Bürkner, P.-C. (2017). Brms: An r package for bayesian multilevel models using stan. Journal of statistical software, $80(1), 1-28$.

Busemeyer, J. R., Gluth, S., Rieskamp, J., \& Turner, B. M. (2019). Cognitive and neural bases of multiattribute, multi-alternative, value-based decisions. Trends in cognitive sciences, 23(3), 251-263.

Calcagnì, A., Lombardi, L., \& Sulpizio, S. (2017). Analyzing spatial data from mouse tracker methodology: An entropic approach. Behavior Research Methods, 49(6), 2012-2030. https://doi.org/10.3758/s13428016-0839-5

Calluso, C., Saulin, A., Baumgartner, T., \& Knoch, D. (2018). Distinct patterns of cognitive conflict dynamics in promise keepers and promise breakers. Frontiers in Psychology, 9. https://doi.org/10.3389/ fpsyg.2018.00939

Cheng, J., \& González-Vallejo, C. (2018). Unpacking decision difficulty: Testing action dynamics in intertemporal, gamble, and consumer choices. Acta psychologica, 190, 199-216.

Christensen, R. H. B. (2019). Ordinal-regression models for ordinal data [R package version 2019.12-10. https://CRAN.R-project.org/package=ordinal].

Cisek, P., \& Kalaska, J. F. (2005). Neural correlates of reaching decisions in dorsal premotor cortex: Specification of multiple direction choices and final selection of action. Neuron, 45(5), 801-814.

Cox, G., Kachergis, G., \& Shiffrin, R. (2012). Gaussian process regression for trajectory analysis. Proceedings of the annual meeting of the cognitive science society, 34(34).

Dale, R., \& Duran, N. D. (2011). The cognitive dynamics of negated sentence verification. Cognitive Science, 35(5), 983-996. https://doi.org/10.1111/j.15516709.2010.01164.x

Dale, R., Kehoe, C., \& Spivey, M. J. (2007). Graded motor responses in the time course of categorizing atypical exemplars. Memory $\mathcal{G}$ Cognition, 35(1), 15-28. https://doi.org/10.3758/BF03195938

Dale, R., Roche, J., Snyder, K., \& McCall, R. (2008). Exploring action dynamics as an index of paired-associate learning. Plos one, 3(3), e1728.

Dotan, D., Pinheiro-Chagas, P., Al Roumi, F., \& Dehaene, S. (2019). Track it to crack it: Dissecting processing stages with finger tracking. Trends in cognitive sciences, 23(12), 1058-1070.
Dshemuchadse, M., Scherbaum, S., \& Goschke, T. (2013). How decisions emerge: Action dynamics in intertemporal decision making. Journal of Experimental Psychology: General, 142(1), 93.

Elliott, D., Hansen, S., Grierson, L. E., Lyons, J., Bennett, S. J., \& Hayes, S. J. (2010). Goal-directed aiming: Two components but multiple processes. Psychological bulletin, 136(6), 1023-1044.

Faulkenberry, T. J., Montgomery, S. A., \& Tennes, S.-A. N. (2015). Response trajectories reveal the temporal dynamics of fraction representations. Acta Psychologica, 159, 100-107.

Faulkenberry, T. J., \& Rey, A. E. (2014). Extending the reach of mousetracking in numerical cognition: A comment on fischer and hartmann (2014). Frontiers in Psychology, 5. https://doi.org/10.3389/fpsyg.2014. 01436

Faulkenberry, T. J., Witte, M., \& Hartmann, M. (2018). Tracking the continuous dynamics of numerical processing: A brief review and editorial. Journal of Numerical Cognition, 4(2), 271-285.

Fernández-Fontelo, A., Kieslich, P. J., Henninger, F., Kreuter, F., \& Greven, S. (2021). Predicting question difficulty in web surveys: A machine learning approach based on mouse movement features. Social Science Computer Review, 08944393211032950.

Fischer, M. H., \& Hartmann, M. (2014). Pushing forward in embodied cognition: May we mouse the mathematical mind? Frontiers in Psychology, 5, 1315. https: //doi.org/10.3389/fpsyg.2014.01315

Forster, K. I. (2004). Category size effects revisited: Frequency and masked priming effects in semantic categorization. Brain and language, 90(1-3), 276-286.

Freeman, J. B. (2014). Abrupt category shifts during realtime person perception. Psychonomic bulletin $\mathcal{E}$ review, 21(1), 85-92.

Freeman, J. B. (2018). Doing psychological science by hand. Current Directions in Psychological Science, 27(5), 315-323.

Freeman, J. B., \& Ambady, N. (2010). MouseTracker: Software for studying real-time mental processing using a computer mouse-tracking method. Behavior Research Methods, 42(1), 226-241. https://doi.org/ 10.3758/BRM.42.1.226

Freeman, J. B., Ambady, N., Rule, N. O., \& Johnson, K. L. (2008). Will a category cue attract you? motor output reveals dynamic competition across person construal. Journal of Experimental Psychology: General, 137(4), 673-690. https://doi.org/10.1037/ a0013875

Freeman, J. B., Nakayama, K., \& Ambady, N. (2013). Finger in flight reveals parallel categorization across mul- 
tiple social dimensions. Social Cognition, 31(6), 792-805.

Friedman, J., Brown, S., \& Finkbeiner, M. (2013). Linking cognitive and reaching trajectories via intermittent movement control. Journal of Mathematical Psychology, 57(3), 140-151. https://doi.org/10.1016/j. jmp.2013.06.005

Frisch, S., Dshemuchadse, M., Görner, M., Goschke, T., \& Scherbaum, S. (2015). Unraveling the subprocesses of selective attention: Insights from dynamic modeling and continuous behavior. Cognitive Processing, 16(4), 377-388. https://doi.org/ 10.1007/s10339-015-0666-0

Fuchs, X., Wulff, D. U., \& Heed, T. (2020). Online sensory feedback during active search improves tactile localization. Journal of Experimental Psychology: Human Perception and Performance, 46(7), 697715 .

Ganor-Stern, D., \& Goldman, R. (2015). Reaching towards an end: Numerical end and distance effects in motor movements. Journal of Cognitive Psychology, 27(4), 490-498.

Gold, J. I., \& Shadlen, M. N. (2001). Neural computations that underlie decisions about sensory stimuli. Trends in cognitive sciences, 5(1), 10-16.

Gold, J. I., \& Shadlen, M. N. (2007). The neural basis of decision making. Annual Review of Neuroscience, 30(1), 535-574. https://doi.org/10.1146/annurev. neuro.29.051605.113038

Grage, T., Schoemann, M., Kieslich, P. J., \& Scherbaum, S. (2019). Lost to translation: How design factors of the mouse-tracking procedure impact the inference from action to cognition. Attention, Perception, $\mathcal{E}$ Psychophysics, 81(7), 2538-2557. https://doi.org/ 10.3758/s13414-019-01889-z

Grosjean, M., Rinkenauer, G., \& Jainta, S. (2012). Where do the eyes really go in the hollow-face illusion? PLOS ONE, 7(9), e44706. https://doi.org/10.1371/journal. pone.0044706

Hartigan, J. A., \& Hartigan, P. M. (1985). The dip test of unimodality. The Annals of Statistics, 13(1), 70-84. https://doi.org/10.1214/aos/1176346577

Haslbeck, J., \& Wulff, D. U. (2020). Estimating the number of clusters via a corrected clustering instability. Computational Statistics, 35, 1879-1894.

Hehman, E., Stolier, R. M., \& Freeman, J. B. (2015). Advanced mouse-tracking analytic techniques for enhancing psychological science. Group Processes $\mathcal{E}$ Intergroup Relations, 18(3), 384-401. https://doi. org/10.1177/1368430214538325

Hennig, C. (2015). What are the true clusters? Pattern Recognition Letters, 64, 53-62.
Henninger, F., \& Kieslich, P. J. (in press). Mousetrap-web: Mouse-tracking for the browser. Behavior Research Methods.

Henninger, F., Shevchenko, Y., Mertens, U. K., Kieslich, P. J., \& Hilbig, B. E. (2021). Lab.js: A free, open, online study builder. Behavior Research Methods, $1-18$.

Hertwig, R., Wulff, D. U., \& Mata, R. (2019). Three gaps and what they may mean for risk preference. Philosophical Transactions of the Royal Society B, 374(1766), 20180140.

Horwitz, R., Kreuter, F., \& Conrad, F. (2017). Using mouse movements to predict web survey response difficulty. Social Science Computer Review, 35(3), 388405.

Incera, S., \& McLennan, C. T. (2016). Mouse tracking reveals that bilinguals behave like experts. Bilingualism, 19(3), 610-620.

Johnson, E. J., Schulte-Mecklenbeck, M., \& Willemsen, M. C. (2008). Process models deserve process data: Comment on Brandstätter, Gigerenzer, and Hertwig (2006). Psychological Review, 115(1), 263272. https://doi.org/10.1037/0033-295X.115.1.263

Kieslich, P. J., \& Henninger, F. (2017). Mousetrap: An integrated, open-source mouse-tracking package. $\mathrm{Be}$ havior Research Methods, 49(5), 1652-1667. https: //doi.org/10.3758/s13428-017-0900-z

Kieslich, P. J., Henninger, F., Wulff, D. U., Haslbeck, J. M. B., \& Schulte-Mecklenbeck, M. (2019). Mouse-tracking: A practical guide to implementation and analysis. In M. Schulte-Mecklenbeck, A. Kühberger, \& J. G. Johnson (Eds.), A handbook of process tracing methods (pp. 111-130). Routledge.

Kieslich, P. J., \& Hilbig, B. E. (2014). Cognitive conflict in social dilemmas: An analysis of response dynamics. Judgment and Decision Making, 9(6), 510-522. Retrieved May 3, 2016, from http://journal.sjdm. org/14/14808/jdm14808.pdf

Kieslich, P. J., Schoemann, M., Grage, T., Hepp, J., \& Scherbaum, S. (2020). Design factors in mousetracking: What makes a difference? Behavior Research Methods, 52(1), 317-341. https://doi.org/10. 3758/s13428-019-01228-y

Koop, G. J., \& Johnson, J. G. (2011). Response dynamics: A new window on the decision process. Judgment $\mathcal{F}$ Decision Making, 6(8).

Koop, G. J., \& Johnson, J. G. (2013). The response dynamics of preferential choice. Cognitive Psychology, 67(4), 151-185. https://doi.org/10.1016/j.cogpsych.2013. 09.001

Krajbich, I., Lu, D., Camerer, C., \& Rangel, A. (2012). The attentional drift-diffusion model extends to simple 
purchasing decisions. Frontiers in psychology, 3, 193.

Lamberts, K. (2000). Information-accumulation theory of speeded categorization. Psychological review, 107(2), 227-260.

Lepora, N. F., \& Pezzulo, G. (2015). Embodied choice: How action influences perceptual decision making. PLOS Computational Biology, 11(4), e1004110. https://doi.org/10.1371/journal.pcbi.1004110

Lopez, R. B., Stillman, P. E., Heatherton, T. F., \& Freeman, J. B. (2018). Minding one's reach (to eat): The promise of computer mouse-tracking to study selfregulation of eating. Frontiers in nutrition, 5, 43.

Martens, M. A., Hasinski, A. E., Andridge, R. R., \& Cunningham, W. A. (2012). Continuous cognitive dynamics of the evaluation of trustworthiness in williams syndrome. Frontiers in psychology, 3, 160.

Martın, J. A. Á., Gollee, H., Müller, J., \& Murray-Smith, R. (2021). Intermittent control as a model of mouse movements. ACM Transactions on ComputerHuman Interaction (TOCHI), 28(5), 1-46.

Mathôt, S., Schreij, D., \& Theeuwes, J. (2012). Opensesame: An open-source, graphical experiment builder for the social sciences. Behavior research methods, 44(2), 314-324.

McKinstry, C., Dale, R., \& Spivey, M. J. (2008). Action dynamics reveal parallel competition in decision making. Psychological Science, 19(1), 22-24.

Miles, J. D., \& Proctor, R. W. (2015). Attention is captured by distractors that uniquely correspond to controlled objects: An analysis of movement trajectories. Attention, Perception, E Psychophysics, 77(3), 819-829.

Moher, J., \& Song, J.-H. (2014). Perceptual decision processes flexibly adapt to avoid change-of-mind motor costs. Journal of vision, 14(8), 1-13.

Moher, J., \& Song, J.-H. (2019). A comparison of simple movement behaviors across three different devices. Attention, Perception, $\mathcal{E}$ Psychophysics, 81(7), 2558-2569. https://doi.org/10.3758/s13414019-01856-8

Murtagh, F., \& Legendre, P. (2014). Ward's hierarchical agglomerative clustering method: Which algorithms implement ward's criterion? Journal of classification, 31(3), 274-295.

Pachur, T., Schulte-Mecklenbeck, M., Murphy, R. O., \& Hertwig, R. (2018). Prospect theory reflects selective allocation of attention. Journal of Experimental Psychology: General, 147(2), 147-169.

Papesh, M. H., \& Goldinger, S. D. (2012). Memory in motion: Movement dynamics reveal memory strength. Psychonomic bulletin $\mathcal{E}$ review, 19(5), 906-913.
Pfister, R., Schwarz, K. A., Janczyk, M., Dale, R., \& Freeman, J. (2013). Good things peak in pairs: A note on the bimodality coefficient. Frontiers in psychology, 4,700 .

Quétard, B., Quinton, J.-C., Colomb, M., Pezzulo, G., Barca, L., Izaute, M., Appadoo, O. K., \& Mermillod, M. (2015). Combined effects of expectations and visual uncertainty upon detection and identification of a target in the fog. Cognitive processing, 16(1), 343348.

R Core Team. (2020). $R$ : A language and environment for statistical computing. R Foundation for Statistical Computing. Vienna, Austria. https :// www . R project.org/

Raaijmakers, J. G., \& Shiffrin, R. M. (1981). Search of associative memory. Psychological review, 88(2), 93.

Resulaj, A., Kiani, R., Wolpert, D. M., \& Shadlen, M. N. (2009). Changes of mind in decision-making. $\mathrm{Na}$ ture, 461(7261), 263-266. https://doi.org/10.1038/ nature 08275

Russo, J. E. (2019). Eye fixations as a process trace. In M. Schulte-Mecklenbeck, A. Kühberger, \& J. G. Johnson (Eds.), A handbook of process tracing methods (pp. 4-26). Routledge.

Scherbaum, S., \& Dshemuchadse, M. (2019). Psychometrics of the continuous mind: Measuring cognitive subprocesses via mouse tracking. Memory $\mathcal{E}$ Cognition, 436-454. https://doi.org/10.3758/s13421019-00981-x

Scherbaum, S., Dshemuchadse, M., Fischer, R., \& Goschke, T. (2010). How decisions evolve: The temporal dynamics of action selection. Cognition, 115(3), 407416. https://doi.org/10.1016/j.cognition.2010.02. 004

Scherbaum, S., Frisch, S., Leiberg, S., Lade, S. J., Goschke, T., \& Dshemuchadse, M. (2016). Process dynamics in delay discounting decisions: An attractor dynamics approach. Judgment and Decision Making, 11(5), 472-495.

Scherbaum, S., \& Kieslich, P. J. (2018). Stuck at the starting line: How the starting procedure influences mousetracking data. Behavior Research Methods, 50(5), 2097-2110. https://doi.org/10.3758/s13428-017$0977-4$

Schoemann, M., Lüken, M., Grage, T., Kieslich, P. J., \& Scherbaum, S. (2019). Validating mouse-tracking: How design factors influence action dynamics in intertemporal decision making. Behavior Research Methods, 51(5), 2356-2377. https://doi .org/10. 3758/s13428-018-1179-4

Schoemann, M., O'Hora, D., Dale, R., \& Scherbaum, S. (2021). Using mouse cursor tracking to investigate online cognition: Preserving methodological inge- 
nuity while moving toward reproducible science. Psychonomic bulletin $\mathcal{F}$ review, 28(3), 766-787.

Schulte-Mecklenbeck, M., \& Huber, O. (2003). Information search in the laboratory and on the web: With or without an experimenter. Behavior Research Methods, Instruments, $\mathcal{F}$ Computers, 35(2), 227-235.

Schulte-Mecklenbeck, M., Johnson, J. G., Böckenholt, U., Goldstein, D. G., Russo, J. E., Sullivan, N. J., \& Willemsen, M. C. (2017). Process-tracing methods in decision making: On growing up in the 70s. Current Directions in Psychological Science, 26(5), 442-450. https : / / doi . org / 10 . 1177/ 0963721417708229

Şimşek, Ö. (2020). Lexicographic decision rule. Oxford research encyclopedia of politics.

Singmann, H., Bolker, B., Westfall, J., \& Aust, F. (2015). Afex: Analysis of factorial experiments. $R$ package version 0.13-145.

Spivey, M. J., Grosjean, M., \& Knoblich, G. (2005). Continuous attraction toward phonological competitors. Proceedings of the National Academy of Sciences of the United States of America, 102(29), 1039310398. https://doi.org/10.1073/pnas.0503903102

Stillerman, B. S., \& Freeman, J. B. (2019). Mouse-tracking to understand real-time dynamics of social cognition. In M. Schulte-Mecklenbeck, A. Kühberger, \& J. G. Johnson (Eds.), A handbook of process tracing methods (pp. 146-160). Routledge.

Stillman, P. E., Shen, X., \& Ferguson, M. J. (2018a). How mouse-tracking can advance social cognitive theory. Trends in cognitive sciences, 22(6), 531-543.

Stillman, P. E., Shen, X., \& Ferguson, M. J. (2018b). How mouse-tracking can advance social cognitive theory. Trends in Cognitive Sciences, 22(6), 531-543. https://doi.org/10.1016/j.tics.2018.03.012

Sullivan, N., Hutcherson, C., Harris, A., \& Rangel, A. (2015). Dietary self-control is related to the speed with which attributes of healthfulness and tastiness are processed. Psychological Science, 26(2), 122134. https://doi.org/10.1177/0956797614559543

Tabatabaeian, M., Dale, R., \& Duran, N. D. (2015). Selfserving dishonest decisions can show facilitated cognitive dynamics. Cognitive Processing, 16(3), 291-300.

Tian, G., \& Wu, W. (2019). A review of mouse-tracking applications in economic studies. Journal of Economics and Behavioral Studies, 11(6 (J)), 1-9.

Tomlinson Jr, J. M., Bailey, T. M., \& Bott, L. (2013). Possibly all of that and then some: Scalar implicatures are understood in two steps. Journal of memory and language, 69(1), 18-35.

van Vugt, F. T., \& Cavanagh, P. (2012). Response trajectories reveal conflict phase in image-word mismatch. At- tention, Perception, $\mathcal{F}$ Psychophysics, 74(2), 263268.

Wickham, H. (2016). ggplot2: Elegant graphics for data analysis. Springer. https://ggplot2.tidyverse.org

Willemsen, M. C., \& Johnson, E. J. (2019). (Re)visiting the decision factory: Observing cognition with mouselabweb. In M. Schulte-Mecklenbeck, A. Kühberger, \& J. G. Johnson (Eds.), A handbook of process tracing methods (pp. 76-95). Routledge.

Wirth, R., Foerster, A., Kunde, W., \& Pfister, R. (2020). Design choices: Empirical recommendations for designing two-dimensional finger-tracking experiments. Behavior Research Methods, 52, 2394-241. https://doi.org/10.3758/s13428-020-01409-0

Wirth, R., Pfister, R., \& Kunde, W. (2016). Asymmetric transfer effects between cognitive and affective task disturbances. Cognition and Emotion, 30(3), 399416. https : // doi . org/10 . 1080/02699931 . 2015. 1009002

Wong, A. L., Haith, A. M., \& Krakauer, J. W. (2015). Motor planning. The Neuroscientist, 21(4), 385-398.

Wulff, D. U., Haslbeck, J. M. B., Kieslich, P. J., Henninger, F., \& Schulte-Mecklenbeck, M. (2019). Mousetracking: Detecting types in movement trajectories. In M. Schulte-Mecklenbeck, A. Kühberger, \& J. G. Johnson (Eds.), A handbook of process tracing methods (pp. 131-145). Routledge.

Wulff, D. U., Haslbeck, J. M. B., \& Schulte-Mecklenbeck, M. (2021). Measuring the (dis-) continuous mind: What movement trajectories reveal about cognition. Manuscript in preparation.

Wulff, D. U., \& Hertwig, R. (2019). Uncovering the anatomy of search without technology. In $\mathrm{M}$. Schulte-Mecklenbeck, A. Kühberger, \& J. G. Johnson (Eds.), A handbook of process tracing methods (pp. 313-325). Routledge.

Wulff, D. U., Hills, T. T., \& Hertwig, R. (2020). Memory is one representation not many: Evidence against wormholes in memory. PsyArXiv. https://doi.org/ 10.31234/osf.io/b5ynj

Wulff, D. U., Mergenthaler-Canseco, M., \& Hertwig, R. (2018). A meta-analytic review of two modes of learning and the description-experience gap. Psychological bulletin, 144(2), 140.

Yentes, J. M., Hunt, N., Schmid, K. K., Kaipust, J. P., McGrath, D., \& Stergiou, N. (2013). The appropriate use of approximate entropy and sample entropy with short data sets. Annals of biomedical engineering, 41(2), 349-365.

Zgonnikov, A., Aleni, A., Piiroinen, P. T., O’Hora, D., \& di Bernardo, M. (2017). Decision landscapes: Visualizing mouse-tracking data. Royal Society open science, 4(11), 170482. 